\title{
Justificatio of the Asymptotic Expansion Method for Homogeneous Isotropic Beams by Comparison with De Saint-Venant's Solutions
}

\author{
Qian Zhao $^{1}$ - Patrice Cartraud ${ }^{1}$ Panagiotis Kotronis ${ }^{1}$
}

\begin{abstract}
The formal asymptotic expansion method is an attractive mean to derive simplifie models for problems exhibiting a small parameter, such as the elastic analysis of beamlike structures. Usually this method is rigorously justifie using convergence theorems $\mathrm{Yu}$ and Hodges, 2004. In this paper it is illustrated how the Saint-Venant's solution naturally arises from the lowest order terms of an asymptotic expansion of the elastic state for the case of homogeneous isotropic beams. It is also highlighted that the Saint-Venant solutions corresponding to pure traction, bending and torsion involve the solution of the first-orde microscopic problems, while for the simple bending problem, the solution of the secondorder microscopic problems is needed. The second-order problems provide therefore a way to characterize the transverse shear behavior and the cross-sectional warping of the beam.
\end{abstract}

Keywords Asymptotic expansion method - Saint-Venant solutions · Transverse shear . Warping $\cdot$ Beam

\section{Introduction}

Beam-like structures are widely used in engineering applications since they exhibit an appealing stiffness and strength to mass ratios. The geometrical feature of these structures is their slenderness, with two dimensions related to the cross-section which have usually the same order of magnitude, much smaller than the third dimension corresponding to the beam

Q. Zhao

qian.zhao@ec-nantes.fr

P. Cartraud

Patrice.Cartraud@ec-nantes.fr

P. Kotronis

Panagiotis.Kotronis@ec-nantes.fr

1 Ecole Centrale de Nantes, Université de Nantes, CNRS, Institut de Recherche en Génie Civil et Mécanique (GeM), 1 Rue de la Noë, 44321 Nantes, France 
length. This feature justifie the large amount of research dedicated to the construction of one-dimensional (1D) beam models.

Mainly two types of approaches can be identifie [21]: the method of hypotheses and asymptotic methods. The method of hypotheses is based on ad hoc assumptions regarding the displacements, strains or stresses distribution over the cross-section. Most of the time these assumptions are expressed under the form of polynomial expansions with respect to cross-section coordinates, but other choices can be made, see [13]. Classical EulerBernoulli's and Timoshenko's theories can thus be obtained from a linear evolution of the displacement across the cross-section. Refine beam models have been proposed adding higher-order terms, see references in $[8,13,18]$. Among them the Carrera Unifie Formulation, which is presented in [8].

The second type of approaches - asymptotic methods - relies on a mathematical and thus more rigorous derivation of the beam model from the three-dimensional (3D) elasticity problem. It exploits the existence of a small parameter corresponding to the inverse of the slenderness ratio. The $3 \mathrm{D}$ elasticity problem then splits in a sequence of local $2 \mathrm{D}$ problems, posed on the cross-section, and 1D problems, which provide the overall response of the structure. The 2D problems are solved for obtaining the overall beam behavior and allows to reconstruct the local 3D response from the 1D solutions. Among these approaches, one can distinguish the variational asymptotic method and the formal asymptotic expansion method. In the variational asymptotic method, as presented in [2] and [11], a warping displacement is introduced and the beam model is constructed from the minimization of the strain energy. One can defin a zero-th order model or higher-order one from the perturbation of the lower-order solution. In the formal asymptotic method $[9,16,21]$ the displacement solution is searched under the form of power series of the small parameter. Again, more or less refine beam models can be define depending on the number of terms of the expansion which are calculated [7]. The objective of this paper is not to compare variational and formal asymptotic methods, which is discussed, e.g., in [14] where both methods are applied on different examples of composite beams.

Whatever the asymptotic method used for constructing the beam model, the question of the justificatio of the model raises. To the authors' knowledge, one of the few articles that address this problem is [22], where the variational asymptotic expansion is applied. However, comparison with Saint-Venant problems is not straightforward. In this paper we provide an alternative way to justify the asymptotic expansion method. For formal asymptotic methods, mathematical results under the form of convergence theorems are available. They state that as the small parameter tends to zero, the $3 \mathrm{D}$ solution converges to the zero-th order asymptotic expansion solution, which turns out to be the Euler-Bernoulli's solution [21]. Other results can also be obtained about the difference between the 3D solution and the asymptotic solution, the latter including higher-order and boundary layer terms, see, e.g., [10] for plates. It has actually to be noticed that the asymptotic solution is valid only in the interior zone of the beam, i.e., far from the edges, because it can not fulfil exactly arbitrary 3D boundary conditions [7]. This makes difficul the comparison between the asymptotic and $3 \mathrm{D}$ solutions.

In this paper, in order to get rid of these edge effects, the Saint-Venant elasticity problems are considered. Thus the $3 \mathrm{D}$ solution of these problems is naturally the interior solution. Moreover, in the case of homogeneous beam made with isotropic material, some analytical solutions are available. This makes possible the direct comparison of the 3D Saint-Venant and asymptotic solutions. The objective of this paper is thus to show that the formal asymptotic solution coincides with the Saint-Venant solution. This constitutes an alternative to convergence theorems [22] for a rigorous justificatio of the formal asymptotic method. 


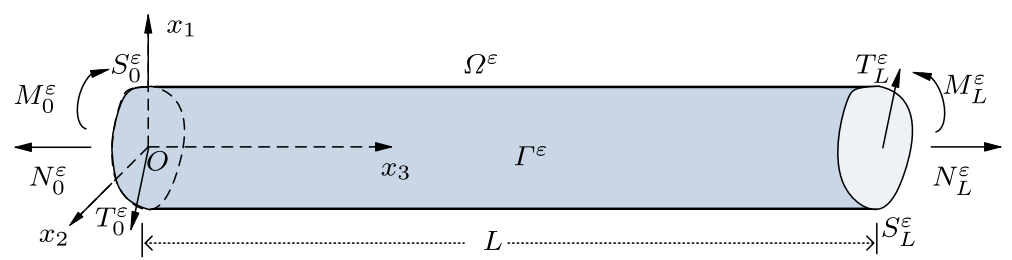

Fig. 1 A prismatic 3D homogeneous isotropic structure with arbitrary cross-section

The outline of this paper is as follows: in Sect. 2 and Sect. 3, the Saint-Venant problems and their solutions for a homogeneous isotropic beam with arbitrary cross-section are presented; in Sect. 4 analytical solutions for the microscopic problems are given. Asymptotic expansion solutions for pure bending and simple bending Saint-Venant problems are then obtained and compared with the Saint-Venant solutions in Sect. 5.

Notation Vectors and higher-order tensors are boldfaced (e.g., $\mathbf{a}^{\varepsilon}$ is the fourth-order 3D elasticity tensor, $\boldsymbol{\sigma}^{\varepsilon}$ the second order stress tensor, $\mathbf{e}^{\varepsilon}$ the second order strain tensor, $\mathbf{u}^{\varepsilon}$ the displacement vector...). Values with subscript ${ }^{\varepsilon}$ usually denote the properties of the 3D structure, while the values without subscript ${ }^{\varepsilon}$ denote the properties of the scaled crosssection, for example, $S^{\varepsilon}$ is the cross-section of the structure, while $S$ denotes the scaled cross-section.

$\operatorname{div}_{\mathbf{x}}$ and $\operatorname{grad}_{\mathbf{x}}^{\mathbf{s}}$ correspond respectively to the divergence and gradient symmetric operator with respect to the coordinate $\mathbf{x}, \mathbf{d i v}_{y_{\alpha}}$ and $\operatorname{grad}_{y_{\alpha}}^{\mathbf{s}}$ are the differential operators with regard to the two microscopic variables $y_{\alpha}$, and $\mathbf{d i v}_{x_{3}} \sigma=\frac{\partial}{\partial x_{3}} \sigma_{i 3} \cdot \mathbf{e}_{i}, \operatorname{grad}_{x_{3}}^{\mathbf{s}}=\operatorname{sym}\left(\frac{\partial \mathbf{u}}{\partial x_{3}} \otimes \mathbf{e}_{3}\right)$. The partial derivatives $\frac{\partial}{\partial x_{3}}, \frac{\partial^{2}}{\partial x_{3}^{2}}$ and $\frac{\partial^{3}}{\partial x_{3}^{3}}$ are denoted as $\partial_{3}, \partial_{33}, \partial_{333}$, and $\frac{\partial^{2}}{\partial x_{i} \partial x_{j}}$ is denoted by $\partial_{i j}^{\varepsilon}$, while $\partial_{i j}$ denotes $\frac{\partial^{2}}{\partial y_{i} \partial y_{j}}$. The Latin indices $i, j, k, l, \ldots$ vary between $1,2,3$ while the Greek indices $\alpha, \beta, \ldots$ from 1,2 . The symbol $\langle\rangle=.\int_{S} . d y_{1} y_{2}$ define the integration over the scaled cross-section. The usual dot product is define as $\mathbf{a} \cdot \mathbf{b}=a_{i} b_{i}$ and the double contraction product as $\mathbf{a}: \mathbf{b}=a_{i j} b_{i j}$. The subscript ${ }^{t}$ define the transverse. $\Delta$ is the Laplace second order differential operator and $\otimes$ the dyadic product.

\section{The 3D Saint-Venant Problem}

Consider a prismatic 3D solid structure with the dimensions of its cross section very small with respect to its length $L$. There exist a small parameter $\varepsilon$ for the description of the structure which denotes the ratio of measure of the cross-section to the axial length of the structure. As shown in Fig. 1, the structure occupies the domain $\Omega^{\varepsilon}$. Consider an orthogonal Cartesian coordinate system $\left(\left(O, x_{1}, x_{2}, x_{3}\right)\right.$, with $\mathbf{e}_{1}, \mathbf{e}_{2}, \mathbf{e}_{3}$ the corresponding unit vectors) with the origin $O$ situated on the surface of the left end cross-section $S_{0}^{\varepsilon}$. The beam axis is along the $x_{3}$ (horizontal) direction. The Cartesian coordinate system is chosen such that:

$$
\int_{S^{\varepsilon}} x_{1} d x_{1} d x_{2}=\int_{S^{\varepsilon}} x_{2} d x_{1} d x_{2}=\int_{S^{\varepsilon}} x_{1} x_{2} d x_{1} d x_{2}=0,
$$

with $S^{\varepsilon}$ the cross-section of the structure. 
The beam boundary is define as $\partial \Omega^{\varepsilon}$ and is composed of the lateral contour $\Gamma^{\varepsilon}$ and the two end sections $S_{0}^{\varepsilon}$ and $S_{L}^{\varepsilon}$ :

$$
\partial \Omega^{\varepsilon}=S_{0}^{\varepsilon} \cup S_{L}^{\varepsilon} \cup \Gamma^{\varepsilon}
$$

The beam is made of a homogeneous and isotropic linear elastic material. The Young's modulus and Poisson's ratio are denoted E and $v$ respectively. Body forces are not considered and the lateral contour $\Gamma^{\varepsilon}$ is traction free. The only loadings are therefore the surface forces at both ends and have the form of a torque $\mathbf{W}^{\varepsilon}$. For example, on the section $S_{L}^{\varepsilon}$ we have:

$$
\mathbf{W}_{L}^{\varepsilon}=\left[T_{1_{L}}^{\varepsilon} \mathbf{e}_{\mathbf{1}}+T_{2_{L}}^{\varepsilon} \mathbf{e}_{2}+N_{L}^{\varepsilon} \mathbf{e}_{\mathbf{3}}, M_{1_{L}}^{\varepsilon} \mathbf{e}_{\mathbf{1}}+M_{2_{L}}^{\varepsilon} \mathbf{e}_{2}+\left(M_{3_{L}}^{\varepsilon}-x_{2 C} T_{1_{L}}^{\varepsilon}+x_{1 C} T_{2_{L}}^{\varepsilon}\right) \mathbf{e}_{3}\right],
$$

where $T_{1_{L}}^{\varepsilon}, T_{2_{L}}^{\varepsilon}$ are the shear forces $\left(T_{1_{L}}^{\varepsilon} \mathbf{e}_{1}+T_{2_{L}}^{\varepsilon} \mathbf{e}_{\mathbf{2}}=\mathbf{T}_{L}^{\varepsilon}\right)$ supposed applied at the shear center of the cross-section with in plane coordinates $\left(x_{1 C}, x_{2 C}\right) . N_{L}^{\varepsilon}$ the axial force and $M_{1_{L}}^{\varepsilon}$, $M_{2_{L}}^{\varepsilon}, M_{3_{L}}^{\varepsilon}$ the moments relative to the surface center $\left(M_{1_{L}}^{\varepsilon} \mathbf{e}_{1}+M_{2_{L}}^{\varepsilon} \mathbf{e}_{\mathbf{2}}=\mathbf{M}_{L}^{\varepsilon}\right)$, define as:

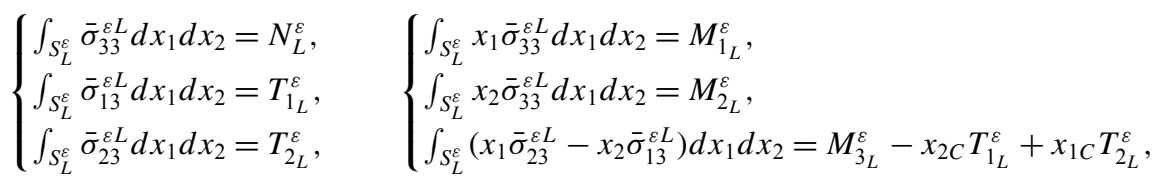

where $\bar{\sigma}_{i j}^{\varepsilon \bullet}$ are stresses at the end section.

Remark The definition above for $M_{1}^{\varepsilon}$ and $M_{2}^{\varepsilon}$ are not the classical ones. They are however introduced to keep consistent with the notation adopted in Sect. 4. The moment of torsion has three components, two are generated by the shear force.

At the end cross-section $S_{0}^{\varepsilon}$, the boundary conditions are similar to Eq. (4):

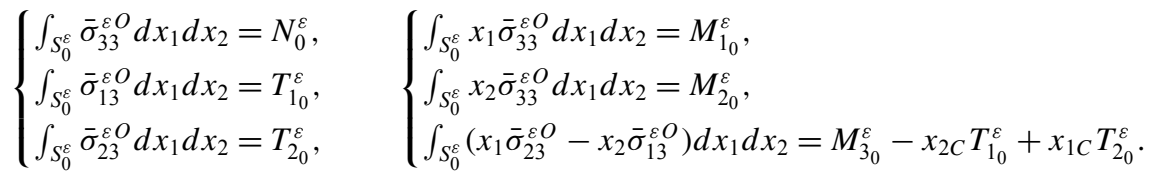

The torques $\mathbf{W}_{0}^{\varepsilon}$ and $\mathbf{W}_{L}^{\varepsilon}$ are such that the overall equilibrium of the structure is satisfied

$$
\left\{\begin{array}{l}
T_{1_{0}}^{\varepsilon}+T_{1_{L}}^{\varepsilon}=0, \\
T_{2_{0}}^{\varepsilon}+T_{2_{L}}^{\varepsilon}=0, \\
N_{0}^{\varepsilon}+N_{L}^{\varepsilon}=0, \\
M_{1_{0}}^{\varepsilon}+M_{1_{L}}^{\varepsilon}+L \cdot T_{1_{L}}^{\varepsilon}=0, \\
M_{2_{0}}^{\varepsilon}+M_{2_{L}}^{\varepsilon}+L \cdot T_{2_{L}}^{\varepsilon}=0, \\
M_{3_{0}}^{\varepsilon}+M_{3_{L}}^{\varepsilon}=0 .
\end{array}\right.
$$


This is a necessary condition for the existence of solutions for the elasticity problem that takes the following form (small perturbation hypothesis):

$$
\begin{cases}\operatorname{div}_{\mathbf{x}} \boldsymbol{\sigma}^{\varepsilon}=\mathbf{0} & \text { in } \Omega^{\varepsilon}, \\ \boldsymbol{\sigma}^{\varepsilon}=\mathbf{a}^{\varepsilon}: \mathbf{e}^{\varepsilon}\left(\mathbf{u}^{\varepsilon}\right) & \text { in } \Omega^{\varepsilon}, \\ \mathbf{e}^{\varepsilon}\left(\mathbf{u}^{\varepsilon}\right)=\operatorname{grad}_{\mathbf{x}}{ }_{\mathbf{s}}\left(\mathbf{u}^{\varepsilon}\right) & \text { in } \Omega^{\varepsilon}, \\ \boldsymbol{\sigma}^{\varepsilon} \cdot \mathbf{n}=\mathbf{0} & \text { on } \Gamma^{\varepsilon},\end{cases}
$$

where $\mathbf{n}$ is the unit normal vector and $\mathbf{0}$ the null vector. For an isotropic material, the coeffi cients $a_{i j k h}$ are expressed as follows:

$$
a_{i j k h}^{\varepsilon}=\lambda \delta_{i j} \delta_{k h}+\mu\left(\delta_{i k} \delta_{j h}+\delta_{i h} \delta_{j k}\right)
$$

with $\lambda$ and $\mu$ the Lamé coefficients

The boundary conditions provided by Eqs. (4) and (5) are given in an integral form, they are not classical (i.e., expressed in terms of stresses) and therefore the solution of the problem of Eqs. (4), (5) and (7) is not unique. However thanks to the Saint-Venant principle [12], two solutions $\boldsymbol{\sigma}^{\varepsilon 1}$ and $\boldsymbol{\sigma}^{\varepsilon 2}$ fulfil the following property: suff ciently far from both ends $\boldsymbol{\sigma}^{\varepsilon 1}-\boldsymbol{\sigma}^{\varepsilon 2} \rightarrow \mathbf{0}$ (the zero tensor), in other words they are identical. A unique solution can thus be define far away from both beam's ends.

\section{The 3D Saint-Venant Solutions}

We recall in this section the elastic solutions of the Saint-Venant problem. They will be used in the following as reference solutions in order to assess the accuracy of the solutions obtained from the asymptotic expansion method.

According to Saint-Venant, the stress solution is searched under the following form:

$$
\left[\begin{array}{ccc}
0 & 0 & \sigma_{13}^{\varepsilon} \\
0 & 0 & \sigma_{23}^{\varepsilon} \\
\sigma_{13}^{\varepsilon} & \sigma_{23}^{\varepsilon} & \sigma_{33}^{\varepsilon}
\end{array}\right],
$$

where $\sigma_{13}^{\varepsilon}, \sigma_{23}^{\varepsilon}, \sigma_{33}^{\varepsilon}$ fulfil the following Beltrami equations [1]:

$$
\left\{\begin{array}{l}
\partial_{11}^{\varepsilon} \sigma_{33}^{\varepsilon}=\partial_{22}^{\varepsilon} \sigma_{33}^{\varepsilon}=\partial_{33}^{\varepsilon} \sigma_{33}^{\varepsilon}=0 \\
\frac{1}{1+\nu} \partial_{13}^{\varepsilon} \sigma_{33}^{\varepsilon}+\Delta^{\varepsilon} \sigma_{13}^{\varepsilon}=0 \\
\frac{1}{1+\nu} \partial_{23}^{\varepsilon} \sigma_{33}^{\varepsilon}+\Delta^{\varepsilon} \sigma_{23}^{\varepsilon}=0 \\
\frac{1}{1+\nu} \partial_{33}^{\varepsilon} \sigma_{33}^{\varepsilon}+\Delta^{\varepsilon} \sigma_{33}^{\varepsilon}=0
\end{array}\right.
$$

The solution of the problem in terms of stresses takes the following form (for more details see [1]):

$$
\left\{\begin{array}{l}
\sigma_{33}^{\varepsilon}=a_{1} x_{1} x_{3}+a_{2} x_{2} x_{3}+a_{3} x_{1}+a_{4} x_{2}+a_{5} x_{3}+a_{6}, \\
\sigma_{13}^{\varepsilon}=\frac{1}{2(1+v)}\left\{\partial_{1}^{\varepsilon} \gamma\left(x_{1}, x_{2}\right)+v \frac{a_{1}}{2}\left(x_{2}^{2}-x_{1}^{2}\right)-v a_{2} x_{1} x_{2}+v c x_{2}\right\} \\
\sigma_{23}^{\varepsilon}=\frac{1}{2(1+v)}\left\{\partial_{2}^{\varepsilon} \gamma\left(x_{1}, x_{2}\right)-v a_{1} x_{1} x_{2}+v \frac{a_{2}}{2}\left(x_{1}^{2}-x_{2}^{2}\right)-v c x_{1}\right\}, \\
\sigma_{11}^{\varepsilon}=\sigma_{22}^{\varepsilon}=\sigma_{12}^{\varepsilon}=0 .
\end{array}\right.
$$


In the above equation:

- $a_{1} a_{2} a_{3} a_{4} a_{5} a_{6}$ are constants identifie using the boundary conditions:

$$
\begin{aligned}
& a_{1}=\frac{T_{1}^{\varepsilon}}{I_{1}^{\varepsilon}}, \quad a_{2}=\frac{T_{2}^{\varepsilon}}{I_{2}^{\varepsilon}}, \quad a_{3}=-\frac{M_{1}^{\varepsilon}+L T_{1}^{\varepsilon}}{I_{1}^{\varepsilon}}, \\
& a_{4}=\frac{M_{2}^{\varepsilon}-L T_{2}^{\varepsilon}}{I_{2}^{\varepsilon}}, \quad a_{5}=0, \quad a_{6}=\frac{N^{\varepsilon}}{\left|S^{\varepsilon}\right|},
\end{aligned}
$$

with $\left|S^{\varepsilon}\right|$ the area of the cross-section $\left(\left|S^{\varepsilon}\right|=\int_{S^{\varepsilon}} d x_{1} x_{2}\right)$ and

$$
I_{\alpha}^{\varepsilon}=\int_{S^{\varepsilon}} x_{\alpha}^{2} d x_{1} x_{2}
$$

As before, the definitio of $I_{\alpha}^{\varepsilon}$ is not the classic one but it is adopted in order to be consistent with the notation used in Sect. 4.

$-\gamma^{\varepsilon}\left(x_{1}, x_{2}\right)$ is the warping function that takes the following form:

$$
\gamma^{\varepsilon}\left(x_{1}, x_{2}\right)=a_{1} \zeta_{1}^{\varepsilon}\left(x_{1}, x_{2}\right)+a_{2} \zeta_{2}^{\varepsilon}\left(x_{1}, x_{2}\right)-v c \varpi^{\varepsilon}\left(x_{1}, x_{2}\right)
$$

corresponding to the following three Neumann problems:

$$
\begin{aligned}
& \begin{cases}\partial_{\alpha \alpha}^{\varepsilon} \zeta_{1}^{\varepsilon}\left(x_{1}, x_{2}\right)=-2 x_{1} & \text { in } S^{\varepsilon}, \\
\partial_{n}^{\varepsilon} \zeta_{1}^{\varepsilon} \cdot n=v\left\{\frac{x_{1}^{2}-x_{2}^{2}}{2} n_{1}+x_{1} x_{2} n_{2}\right\} & \text { on } \partial S^{\varepsilon}, \\
\int_{S^{\varepsilon}} \zeta_{1}^{\varepsilon} d S^{\varepsilon}=0, & \text { in } S^{\varepsilon},\end{cases} \\
& \begin{cases}\partial_{\alpha \alpha}^{\varepsilon} \zeta_{2}^{\varepsilon}\left(x_{1}, x_{2}\right)=-2 x_{2} & \text { on } \partial S^{\varepsilon}, \\
\partial_{n}^{\varepsilon} \zeta_{2}^{\varepsilon} \cdot n=v\left\{x_{1} x_{2} n_{1}-\frac{x_{1}^{2}-x_{2}^{2}}{2} n_{2}\right\} & \\
\int_{S^{\varepsilon}} \zeta_{2}^{\varepsilon} d S^{\varepsilon}=0, & \text { in } S^{\varepsilon},\end{cases} \\
& \begin{cases}\partial_{\alpha \alpha}^{\varepsilon} \varpi^{\varepsilon}\left(x_{1}, x_{2}\right)=0 & \text { on } \partial S^{\varepsilon}, \\
\partial_{n}^{\varepsilon} \varpi^{\varepsilon} \cdot n=x_{2} n_{1}-x_{1} n_{2} & \\
\int_{S^{\varepsilon}} \varpi^{\varepsilon} d S^{\varepsilon}=0, & \end{cases}
\end{aligned}
$$

where $\zeta_{1}^{\varepsilon}$ and $\zeta_{2}^{\varepsilon}$ are the shear warping functions and $\varpi^{\varepsilon}$ the torsion warping function. It can be easily checked that the above three problems are well-posed, with $\zeta_{1}^{\varepsilon}, \zeta_{2}^{\varepsilon}$ and $\varpi^{\varepsilon}$ unique because their average value over the cross-section is 0 .

$-c$ is define as:

$$
\frac{v}{2(1+v)} J^{\varepsilon} \cdot c=T_{1}^{\varepsilon} x_{2 C}-T_{2}^{\varepsilon} x_{1 C}-M_{3}^{\varepsilon},
$$

with $J^{\varepsilon}$ the polar moment of inertia define as:

$$
J^{\varepsilon}=I_{1}^{\varepsilon}+I_{2}^{\varepsilon}-\int_{S^{\varepsilon}}\left[\left(\partial_{1}^{\varepsilon} \varpi^{\varepsilon}\right)^{2}+\left(\partial_{2}^{\varepsilon} \varpi^{\varepsilon}\right)^{2}\right] d x_{1} x_{2} .
$$

As mentioned before and according to Eq. (16), torsion is therefore composed of three components for the general case of a beam with arbitrary cross-sections: one due to the torque and the other two due to shear forces. 


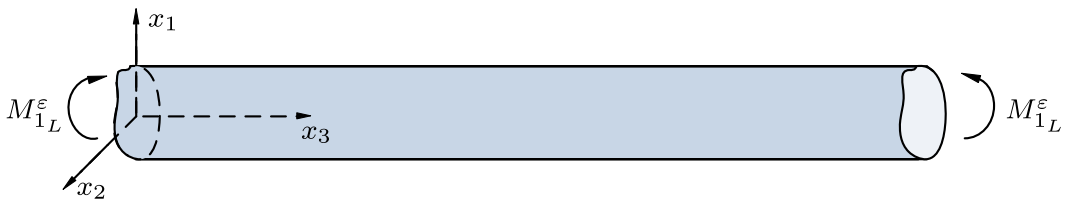

Fig. 2 Loadings applied on the prism (pure bending)

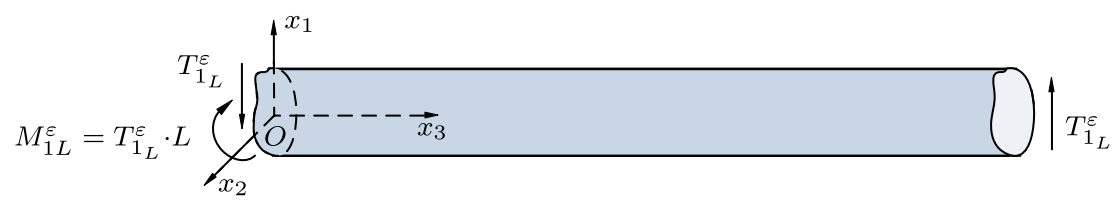

Fig. 3 Loadings applied on the prism (simple bending)

The point $C$ with coordinates $\left(x_{1 C}, x_{2}\right)$ is the shear center of the cross section. Its position depends on the geometry of the section and the Poisson's ratio [1]. For a beam with a symmetric plane, the point $C$ is located on that plane. For a beam cross-section with two symmetric planes, $C$ coincides with the surface center.

The stress define in Eqs. (11)-(16) is thus a solution of the problem Eqs. (4), (5) and (7), and is unique far away from the beam's ends. The 3D Saint-Venant solutions for two specifi loading cases are presented hereafter: the pure bending and the simple bending problems.

\subsection{Saint-Venant Solution for the Pure Bending Problem}

Consider the pure bending problem shown in Fig. 2, where the torque applied on the two end sections reduces to a moment $M_{1_{L}}^{\varepsilon}$ on $S_{L}^{\varepsilon}$ (that is $T_{1_{L}}^{\varepsilon}=T_{2_{L}}^{\varepsilon}=N_{L}^{\varepsilon}=M_{2_{L}}^{\varepsilon}=M_{3_{L}}^{\varepsilon}=0$ ) and $-M_{1_{L}}^{\varepsilon}$ on $S_{0}^{\varepsilon}$ due to the overall equilibrium. In that case, due to Eqs. (11) and (12) the Saint-Venant solution corresponds to a 1D stress state with:

$$
\sigma_{33}^{\varepsilon}=-\frac{M_{1_{L}}^{\varepsilon}}{I_{1}^{\varepsilon}} x_{1} .
$$

\subsection{Saint-Venant Solution for the Simple Bending Problem}

In Fig. 3, the non zero loads are: $T_{1_{L}}^{\varepsilon}$ on the right end section $S_{L}^{\varepsilon}, T_{1_{L}}^{\varepsilon}$ and $\left(T_{1_{L}}^{\varepsilon} \cdot L\right)$ on the left end section $S_{0}^{\varepsilon}$. The constants in Eqs. (12) and (16) are now given by:

$$
\begin{aligned}
& a_{1}=\frac{T_{1_{L}}^{\varepsilon}}{I_{1}^{\varepsilon}}, \quad a_{2}=0, \quad a_{3}=-\frac{L \cdot T_{1_{L}}^{\varepsilon}}{I_{1}^{\varepsilon}}, \quad a_{4}=0, \\
& a_{5}=0, \quad a_{6}=0, \quad c=\frac{T_{1_{L}}^{\varepsilon} \cdot x_{2 C}}{J^{\varepsilon}} \cdot \frac{2(1+v)}{v} .
\end{aligned}
$$

According to Eq. (11), the axial stress $\sigma_{33}^{\varepsilon}$ becomes:

$$
\sigma_{33}^{\varepsilon}=\frac{T_{1_{L}}^{\varepsilon}}{I_{1}^{\varepsilon}} x_{1}\left(x_{3}-L\right) .
$$


Following Eq. (14) and considering that $a_{2}=0$ the warping function is:

$$
\gamma^{\varepsilon}\left(x_{1}, x_{2}\right)=\frac{T_{1_{L}}^{\varepsilon}}{I_{1}^{\varepsilon}} \zeta_{1}^{\varepsilon}\left(x_{1}, x_{2}\right)-2(1+v) \frac{T_{1_{L}}^{\varepsilon} \cdot x_{2 C}}{J^{\varepsilon}} \cdot \varpi^{\varepsilon}\left(x_{1}, x_{2}\right),
$$

and the shear stresses $\sigma_{13}^{\varepsilon}$ and $\sigma_{23}^{\varepsilon}$ are obtained from Eq. (11):

$$
\left\{\begin{array}{l}
\sigma_{13}^{\varepsilon}=\frac{1}{2(1+v)} \cdot \frac{T_{1_{L}}^{\varepsilon}}{I_{1}^{\varepsilon}}\left[\frac{1}{2} v\left(x_{2}^{2}-x_{1}^{2}\right)+\partial_{1}^{\varepsilon} \zeta_{1}^{\varepsilon}\left(x_{1}, x_{2}\right)\right]-\frac{T_{1_{L}}^{\varepsilon} \cdot x_{2 C}}{J^{\varepsilon}} \cdot\left[\partial_{1}^{\varepsilon} \varpi^{\varepsilon}\left(x_{1}, x_{2}\right)-x_{2}\right], \\
\sigma_{23}^{\varepsilon}=\frac{1}{2(1+v)} \frac{T_{1_{L}}^{\varepsilon}}{I_{1}^{\varepsilon}}\left[-v x_{1} x_{2}+\partial_{2}^{\varepsilon} \zeta_{1}^{\varepsilon}\left(x_{1}, x_{2}\right)\right]-\frac{T_{1_{L}}^{\varepsilon} \cdot x_{2 C}}{J^{\varepsilon}} \cdot\left[\partial_{2}^{\varepsilon} \varpi^{\varepsilon}\left(x_{1}, x_{2}\right)+x_{1}\right]
\end{array}\right.
$$

with $\zeta_{1}^{\varepsilon}\left(x_{1}, x_{2}\right), \varpi^{\varepsilon}\left(x_{1}, x_{2}\right)$ solutions of the problems define by Eq. (15).

- For the specifi case where no torsion is generated by the shear force $(c=0)$, the previous equations are simplified

$$
\left\{\begin{array}{l}
\sigma_{13}^{\varepsilon,(c=0)}=\frac{1}{2(1+v)}\left[\frac{1}{2} v\left(x_{2}^{2}-x_{1}^{2}\right)+\partial_{1}^{\varepsilon} \zeta_{1}^{\varepsilon}\left(x_{1}, x_{2}\right)\right] \frac{T_{1_{L}}^{\varepsilon}}{I_{1}^{\varepsilon}} \\
\sigma_{23}^{\varepsilon,(c=0)}=\frac{1}{2(1+v)}\left[-v x_{1} x_{2}+\partial_{2}^{\varepsilon} \zeta_{1}^{\varepsilon}\left(x_{1}, x_{2}\right)\right] \frac{T_{1}^{\varepsilon}}{I_{1}^{\varepsilon}}
\end{array}\right.
$$

For the particular case of a beam with a circular cross-section with a radius $\mathrm{R}$, $\zeta_{1}^{\varepsilon}\left(x_{1}, x_{2}\right)$ has the following analytical expression [1]:

$$
\zeta_{1}^{\varepsilon}\left(x_{1}, x_{2}\right)=\left(\frac{v}{2}+\frac{3}{4}\right) R^{2} x_{1}-\frac{1}{4}\left(x_{1}^{3}+x_{2}^{2} x_{1}\right),
$$

which leads to the following expressions:

$$
\left\{\begin{array}{l}
\sigma_{13}^{\varepsilon,(\text { circular })}=-\frac{1}{8(1+v) I_{1}^{\varepsilon}} T_{1_{L}}^{\varepsilon}\left[(3+2 v)\left(x_{1}^{2}-R^{2}\right)+(1-2 v) x_{2}^{2}\right] \\
\sigma_{23}^{\varepsilon,(\text { circular })}=-\frac{1+2 v}{4(1+v) I_{1}^{\varepsilon}} T_{1_{L}}^{\varepsilon} x_{1} x_{2}
\end{array}\right.
$$

\section{The Asymptotic Expansion Method}

In this section, the 3D Saint-Venant problem is again considered but it is this time solved with the asymptotic expansion method. It will be shown that the corresponding solutions coincide with the exact solutions obtained in the framework of 3D elasticity (see Sect. 3).

In order to use asymptotic expansion techniques, two scales are introduced thanks to the small parameter $\varepsilon$ which equals the inverse of the slenderness ratio following the transformation rules:

$$
y_{\alpha}=x_{\alpha} / \varepsilon, \quad x_{3}=x_{3} .
$$

Therefore the initial 3D problem Eqs. (4),(5) and (7) splits in two scales: macroscopic 1D problems in the $x_{3}$ direction and microscopic 2D problems on the scaled cross-section $S$ (plane $y_{1} O y_{2}$ ). The following steps are performed:

(1) The starting point of the asymptotic expansion method is the assumption that the displacement field solution of the 3D Saint-Venant problem, has the following form [20] (with $\mathbf{e}_{\alpha}$ the unit vectors on the scaled cross-section $S$ ):

$$
\mathbf{u}^{\varepsilon}\left(y_{\beta}, x_{3}\right)=\hat{u}_{\alpha}^{0}\left(x_{3}\right) \mathbf{e}_{\alpha}+\varepsilon \mathbf{u}^{1}\left(y_{\beta}, x_{3}\right)+\varepsilon^{2} \mathbf{u}^{2}\left(y_{\beta}, x_{3}\right)+\cdots,
$$


the following notation is hereafter adopted:

- $\mathbf{u}^{p}=\left(u_{1}^{p}, u_{2}^{p}, u_{3}^{p}\right), p \geq 0$, where $u_{i}^{p}$ denotes the $i$ direction component of the order $p$ displacement field

- $\mathbf{u}^{p}\left(y_{\beta}, x_{3}\right)=\mathbf{u}^{p}\left(y_{1}, y_{2}, x_{3}\right)$ with $\left(y_{1}, y_{2}, x_{3}\right)=\left(\frac{x_{1}}{\varepsilon}, \frac{x_{2}}{\varepsilon}, x_{3}\right)$, (see Eq. (26)).

(2) Following Eq. (26), one has the derivation rule:

$$
\frac{\partial}{\partial x_{\alpha}}=\frac{1}{\varepsilon} \frac{\partial}{\partial y_{\alpha}}
$$

(3) The elasticity moduli $a_{i j k l}^{\varepsilon}$ are assumed to be independent of $\varepsilon$, and so we can write that: $\mathbf{a}^{\varepsilon}\left(x_{\beta}\right)=\mathbf{a}\left(y_{\beta}\right)$.

Combining the displacement f eld Eq. (27) and the derivation rule Eq. (28) the asymptotic expansions for the strain and stress become:

$$
\left\{\begin{array}{l}
\mathbf{e}^{\varepsilon}\left(y_{\beta}, x_{3}\right)=\mathbf{e}^{0}\left(y_{\beta}, x_{3}\right)+\varepsilon \mathbf{e}^{1}\left(y_{\beta}, x_{3}\right)+\varepsilon^{2} \mathbf{e}^{2}\left(y_{\beta}, x_{3}\right)+\cdots, \\
\text { with } \mathbf{e}^{p}\left(y_{\beta}, x_{3}\right)=\mathbf{e}_{y_{\alpha}}\left(\mathbf{u}^{p+1}\right)+\mathbf{e}_{x_{3}}\left(\mathbf{u}^{p}\right), \\
\text { and }\left\{\begin{array}{l}
\mathbf{e}_{y_{\alpha}}\left(\mathbf{u}^{p+1}\right)=\operatorname{grad}_{y_{\alpha}}^{\mathbf{s}}\left(\mathbf{u}^{p+1}\right), \quad \forall p \geq 0, \\
\mathbf{e}_{x_{3}}\left(\mathbf{u}^{p}\right)=\operatorname{grad}_{x_{3}}^{\mathbf{s}}\left(\mathbf{u}^{p}\right),
\end{array}\right.
\end{array}\right.
$$

and

$$
\left\{\begin{array}{c}
\boldsymbol{\sigma}^{\varepsilon}\left(y_{\beta}, x_{3}\right)=\boldsymbol{\sigma}^{0}\left(y_{\beta}, x_{3}\right)+\varepsilon \boldsymbol{\sigma}^{1}\left(y_{\beta}, x_{3}\right)+\varepsilon^{2} \boldsymbol{\sigma}^{2}\left(y_{\beta}, x_{3}\right)+\cdots, \\
\text { with } \boldsymbol{\sigma}^{p}\left(y_{\beta}, x_{3}\right)=\mathbf{a}\left(y_{\beta}\right): \mathbf{e}^{p}\left(y_{\beta}, x_{3}\right), \quad \forall p \geq 0 .
\end{array}\right.
$$

(4) As already mentioned, the beam is assumed to be free of body forces and surface tractions on the lateral boundary $\Gamma^{\varepsilon}$. By applying the divergence to the stress fiel Eq. (30), the equilibrium equation takes thus the following form:

$$
\varepsilon^{-1} \operatorname{div}_{y_{\alpha}} \boldsymbol{\sigma}^{0}+\sum_{p \geq 0} \varepsilon^{p}\left(\operatorname{div}_{x_{3}} \boldsymbol{\sigma}^{p}+\operatorname{div}_{y_{\alpha}} \boldsymbol{\sigma}^{p+1}\right)=\mathbf{0} .
$$

A series of microscopic 2D problems and macroscopic 1D problems can now be identified each corresponding to a specifi order of $\varepsilon$ in the asymptotic expansion [20]. Specifi solutions are presented in the next section.

The relations between the variables define on the macroscopic and microscopic scales are found hereafter. The bending moment is define as:

$$
M_{\alpha}^{\varepsilon}\left(y_{\beta}, x_{3}\right)=\int_{S^{\varepsilon}} x_{\alpha} \sigma_{33}^{\varepsilon}\left(y_{\beta}, x_{3}\right) d x_{1} d x_{2} .
$$

Due to Eq. (26) and the asymptotic expansion of $\sigma_{33}^{\varepsilon}\left(y_{\beta}, x_{3}\right)$ in Eq. (30), we can also defin $M_{\alpha}^{\varepsilon}\left(x_{3}\right)$ using microscopic scale variables (on the scaled cross-section $S$ ):

$$
\begin{aligned}
M_{\alpha}^{\varepsilon}\left(y_{\beta}, x_{3}\right) & =\varepsilon^{2} \int_{S} x_{\alpha} \sigma_{33}^{\varepsilon}\left(y_{\beta}, x_{3}\right) d y_{1} d y_{2} \\
& =\varepsilon^{3} \int_{S} y_{\alpha}\left[\sigma_{33}^{0}\left(y_{\beta}, x_{3}\right)+\varepsilon \sigma_{33}^{1}\left(y_{\beta}, x_{3}\right)+\varepsilon^{2} \sigma_{33}^{2}\left(y_{\beta}, x_{3}\right)+\cdots\right] d y_{1} d y_{2} .
\end{aligned}
$$


In Eq. (33) if we consider that $\int_{S}-y_{\alpha} \sigma_{33}^{k}\left(y_{\alpha}, x_{3}\right) d y_{1} d y_{2}=M_{\alpha}^{k}\left(x_{3}\right)$, the relation between $M_{\alpha}^{\varepsilon}\left(y_{\beta}, x_{3}\right)$ over the original cross-section $S^{\varepsilon}$ and $M_{\alpha}^{0}\left(x_{3}\right), M_{\alpha}^{1}\left(x_{3}\right), \ldots$ on the scaled crosssection $S$ is thus given by:

$$
M_{\alpha}^{\varepsilon}\left(y_{\beta}, x_{3}\right)=\varepsilon^{3}\left[M_{\alpha}^{0}\left(x_{3}\right)+\varepsilon M_{\alpha}^{1}\left(x_{3}\right)+\varepsilon^{2} M_{\alpha}^{2}\left(x_{3}\right)+\cdots\right] .
$$

Equation (34) shows that the bending moment of the original 3D structure $M_{\alpha}^{\varepsilon}\left(y_{\beta}, x_{3}\right)$ and $\sum_{k \geq 0} M_{\alpha}^{k}\left(x_{3}\right)$ are not of the same order. In other words, $\sum_{k \geq 0} M_{\alpha}^{k}\left(x_{3}\right)$ does not represent the bending moment of the original 3D structure, but equals the bending moment of the original $3 \mathrm{D}$ structure at $k+3$ order.

Using the same approach, the following equations are found:

$$
\left\{\begin{array}{l}
N^{\varepsilon}\left(y_{\beta}, x_{3}\right)=\varepsilon^{2}\left(N^{0}\left(x_{3}\right)+\varepsilon N^{1}\left(x_{3}\right)+\varepsilon^{2} N^{2}\left(x_{3}\right)+\cdots\right), \\
T_{\alpha}^{\varepsilon}\left(y_{\beta}, x_{3}\right)=\varepsilon^{2}\left(T_{\alpha}^{0}\left(x_{3}\right)+\varepsilon T_{\alpha}^{1}\left(x_{3}\right)+\varepsilon^{2} T_{\alpha}^{2}\left(x_{3}\right)+\cdots\right), \\
M_{\alpha}^{\varepsilon}\left(y_{\beta}, x_{3}\right)=\varepsilon^{3}\left(M_{\alpha}^{0}\left(x_{3}\right)+\varepsilon M_{\alpha}^{1}\left(x_{3}\right)+\varepsilon^{2} M_{\alpha}^{2}\left(x_{3}\right)+\cdots\right), \\
M_{3}^{\varepsilon}\left(y_{\beta}, x_{3}\right)=\varepsilon^{3}\left(M_{3}^{0}\left(x_{3}\right)+\varepsilon M_{3}^{1}\left(x_{3}\right)+\varepsilon^{2} M_{3}^{2}\left(x_{3}\right)+\cdots\right) .
\end{array}\right.
$$

Equation (35) is used to f nd the order of loadings applied on the scaled cross-section, with:

$$
\left\{\begin{array}{l}
N^{k}\left(x_{3}\right)=\left\langle\sigma_{33}^{k}\left(y_{\beta}, x_{3}\right)\right\rangle \\
T_{\alpha}^{k}\left(x_{3}\right)=\left\langle\sigma_{\alpha 3}^{k}\left(y_{\beta}, x_{3}\right)\right\rangle \\
M_{\alpha}^{k}\left(x_{3}\right)=\left\langle-y_{\alpha} \sigma_{33}^{k}\left(y_{\beta}, x_{3}\right)\right\rangle \\
M_{3}^{k}\left(x_{3}\right)=\left\langle\left(-y_{2} \sigma_{13}^{k}\left(y_{\beta}, x_{3}\right)+y_{1} \sigma_{23}^{k}\left(y_{\beta}, x_{3}\right)\right)\right\rangle
\end{array}\right.
$$

Boundary conditions at each order The boundary conditions for the initial 3D problem have been presented in Eq. (3): they are expressed under the form of a torque $\mathbf{W}^{\varepsilon}$.

In [20], the authors showed that bending moments applied on the scaled end cross-section (for example on $S_{L}$ ) $M_{\alpha}^{\prime}$ are $\varepsilon^{-4}$ order of the bending moments applied on the original 3D structure $M_{\alpha L}^{\varepsilon}$ :

$$
M_{\alpha L}^{\varepsilon}=\varepsilon^{4} M_{\alpha L}^{\prime}
$$

This condition is required in order that the solution of the $3 \mathrm{D}$ elastic problem satisfie the small displacements and small strains assumption, see [17, 19, 20]. In the same way, it can be shown that for the other components of the torque at the end sections of the beam, one has:

$$
\left\{\begin{array}{l}
N_{L}^{\varepsilon}=\varepsilon^{3} N_{L}^{\prime}, \\
T_{\alpha_{L}}^{\varepsilon}=\varepsilon^{4} T_{\alpha_{L}}^{\prime}, \\
M_{\alpha_{L}}^{\varepsilon}=\varepsilon^{4} M_{\alpha_{L}}^{\prime}, \\
M_{3_{L}}^{\varepsilon}-x_{2 C} T_{1_{L}}^{\varepsilon}+x_{1 C} T_{2_{L}}^{\varepsilon}=\varepsilon^{4} M_{3_{L}}^{\prime}-\varepsilon^{5} y_{2 C} T_{1_{L}}^{\prime}+\varepsilon^{5} y_{1 C} T_{2_{L}}^{\prime} .
\end{array}\right.
$$

The same relations are valid for the left end section $S_{0}^{\varepsilon}$ thanks to the overall equilibrium. Eq. (38) provides the relations between the initial 3D boundary conditions $\left(N_{L}^{\varepsilon}, T_{\alpha L}^{\varepsilon}, M_{\alpha L}^{\varepsilon}\right.$ and $\left.M_{3 L}^{\varepsilon}\right)$, and the ones define on the scaled cross-section $\left(N_{L}^{\prime}, T_{\alpha L}^{\prime}, M_{\alpha L}^{\prime}\right.$ and $\left.M_{3 L}^{\prime}\right)$. At 
boundaries (for example $S_{L}$ ), by identifying the terms of the same order in Eq. (38) and Eq. (35), the following equations can be found:

$$
\left\{\begin{array} { l } 
{ N ^ { 1 } ( L ) = N _ { L } ^ { \prime } , } \\
{ T _ { \alpha } ^ { 1 } ( L ) = 0 , } \\
{ M _ { \alpha } ^ { 1 } ( L ) = M _ { \alpha _ { L } } ^ { \prime } , } \\
{ M _ { 3 } ^ { 1 } ( L ) = M _ { 3 _ { L } } ^ { \prime } , }
\end{array} \quad \left\{\begin{array} { l } 
{ N ^ { 2 } ( L ) = 0 , } \\
{ T _ { \alpha } ^ { 2 } ( L ) = T _ { \alpha _ { L } } ^ { \prime } , } \\
{ M _ { \alpha } ^ { 2 } ( L ) = 0 , } \\
{ M _ { 3 } ^ { 2 } ( L ) = - y _ { 2 C } T _ { 1 _ { L } } ^ { \prime } + y _ { 1 C } T _ { 2 _ { L } } ^ { \prime } , }
\end{array} \quad \left\{\begin{array}{l}
N^{k}(L)=0, \\
T_{\alpha}^{k}(L)=0, \quad k \geq 3 . \\
M_{\alpha}^{k}(L)=0, \\
M_{3}^{k}(L)=0,
\end{array}\right.\right.\right.
$$

From Eq. (39), one can conclude that in the asymptotic expansion method, extension, bending and torsion appear at the firs order; shear forces and torsion moment caused by the shear forces appear at the second order.

\subsection{Microscopic 2D Problems: $P_{2 D}^{k}$}

The $P_{2 D}^{k}$ problem at $\varepsilon^{k}$ order is define by identifying the $k$ th order term of the equilibrium equation Eq. (31), completing the constitutive equation by Eqs. (29) and (30), and considering the boundary conditions on $\partial S$. It consists in findin $\boldsymbol{\sigma}^{k+1}\left(y_{\beta}, x_{3}\right), \mathbf{e}^{k+1}\left(y_{\beta}, x_{3}\right)$ and $\mathbf{u}^{k+2}\left(y_{\beta}, x_{3}\right)$ that satisfy the following equations:

$$
P_{2 D}^{k} \begin{cases}\operatorname{div}_{y_{\alpha}} \boldsymbol{\sigma}^{k+1}=-\mathbf{d i v}_{x_{3}} \boldsymbol{\sigma}^{k} & \text { in } S, \\ \boldsymbol{\sigma}^{k+1}=\mathbf{a}\left(y_{\beta}\right): \mathbf{e}^{k+1} & \text { in } S, \\ \mathbf{e}^{k+1}=\mathbf{e}_{y_{\alpha}}\left(\mathbf{u}^{k+2}\right)+\mathbf{e}_{x_{3}}\left(\mathbf{u}^{k+1}\right) & \text { in } S, \\ \boldsymbol{\sigma}^{k+1} \cdot \mathbf{n}=\mathbf{0} & \text { on } \partial S,\end{cases}
$$

with $k \geq-1$ and $\sigma^{-1}=\mathbf{e}^{-1}=0$.

Solving the problem $P_{2 D}^{k}$ is based on the fact that the problems at previous orders have already been solved, and therefore $\boldsymbol{\sigma}^{k}$ and $\mathbf{u}^{k+1}$ are data of the problem.

The variational form of problem Eq. (40) consists in findin the feld $\mathbf{u}^{k+2}$ such that:

$$
\forall \psi, \quad \int_{S} \boldsymbol{\sigma}^{k+1}: \mathbf{e}_{y_{\alpha}}(\psi) d S=\int_{S} \operatorname{div}_{x_{3}} \boldsymbol{\sigma}^{k} \cdot \psi d S
$$

Due to the variational form Eq. (41), the existence of a solution for the $P_{2 D}^{k}$ problem is guaranteed if $\operatorname{div}_{x_{3}} \boldsymbol{\sigma}^{k}$, which plays the role of a body load, is such that:

$$
\forall \mathbf{v} \in \mathcal{R} \quad \int_{S} \operatorname{div}_{x_{3}} \sigma^{k} \cdot \mathbf{v} d S=0
$$

where $\mathcal{R}$ corresponds to a set of rigid body motions formed by arbitrary translation $\hat{v}_{i}\left(x_{3}\right)$ and rotation of the axis $x_{3} \varphi\left(x_{3}\right)$, as follows:

$$
\mathcal{R}=\left\{\mathbf{v}\left(y_{\alpha}, x_{3}\right) / \mathbf{v}=\hat{v}_{i}\left(x_{3}\right) \cdot \mathbf{e}_{\mathbf{i}}+\varphi\left(x_{3}\right)\left[y_{1} \cdot \mathbf{e}_{2}-y_{2} \cdot \mathbf{e}_{1}\right]\right\} .
$$

Under this necessary condition Eq. (42), the solutions $\boldsymbol{\sigma}^{k+1}, \mathbf{e}^{k+1}$ and $\mathbf{u}^{k+2}$ (determined up to an element of $\mathcal{R}$ ) exist and can be linearly expressed with respect to the data contained in $\mathbf{d i v}_{x_{3}} \boldsymbol{\sigma}^{k}$ and $\mathbf{e}_{x_{3}}\left(\mathbf{u}^{k+1}\right)$. As presented in [20,21] and [6], the compatibility condition Eq. (42) enables to formulate the macroscopic problems, as shown in the next section. 


\subsubsection{Microscopic Problem at $\varepsilon^{-1}$ Order: $P_{2 D}^{-1}$}

The data of the problem at this order is $\mathbf{u}^{0}\left(x_{3}\right)$ (see its form in Eq. (27)), which appears through $\mathbf{e}_{x_{3}}\left(\mathbf{u}^{0}\right)$. The problem is well posed because the body load is zero. It can be easily established that $\mathbf{u}_{\text {part }}^{1}=-y_{\alpha} \partial_{3} \hat{u}_{\alpha}^{0}\left(x_{3}\right) \mathbf{e}_{3}$ and $\boldsymbol{\sigma}_{\text {part }}^{0}=\mathbf{e}_{\text {part }}^{0}=\mathbf{0}$ is a solution of the problem (where the subscript part refers to 'particular', since as it can be seen the solution is not unique).

The displacement solution $\mathbf{u}_{\text {part }}^{1}$ being define up to an element of $\mathcal{R}$, the general form of the solution at this order is [20]:

$$
\left\{\begin{aligned}
\mathbf{u}^{1} & =\hat{u}_{i}^{1}\left(x_{3}\right) \mathbf{e}_{\mathbf{i}}+\varphi^{1}\left(x_{3}\right)\left[y_{1} \mathbf{e}_{2}-y_{2} \mathbf{e}_{1}\right]-y_{\alpha} \partial_{3} \hat{u}_{\alpha}^{0}\left(x_{3}\right) \mathbf{e}_{3} \\
& \equiv \mathbf{u}^{1}\left(y_{\beta}, x_{3}\right)
\end{aligned} \text { and } \quad \boldsymbol{\sigma}^{0}=\mathbf{e}^{0}=\mathbf{0} .\right.
$$

A displacement fiel of this type (the sum of the trivial solution of the cellular problem and the element of $\mathcal{R}$ ) will be found at each order and it is hereafter noted as $\mathbf{u}^{k}\left(y_{\beta}, x_{3}\right)$.

So far the displacement fiel $\mathbf{u}^{\varepsilon}$ is of the form:

$$
\mathbf{u}^{\varepsilon} \simeq\left\{\begin{array}{c}
\hat{u}_{1}^{0}\left(x_{3}\right)-x_{2} \varphi^{1}\left(x_{3}\right) \\
\hat{u}_{2}^{0}\left(x_{3}\right)+x_{1} \varphi^{1}\left(x_{3}\right) \\
-x_{\alpha} \partial_{3} \hat{u}_{\alpha}^{0}\left(x_{3}\right)+\varepsilon \hat{u}_{3}^{1}\left(x_{3}\right)
\end{array}\right\}
$$

It is easy to $\mathrm{f}$ nd that the form of this displacement $\mathrm{f}$ eld corresponds to the Bernoulli theory hypothesis: plane sections remain plane and normal to the axis of the beam, under a translation $\hat{u}_{\alpha}^{0}\left(x_{3}\right) \mathbf{e}_{\alpha}+\varepsilon \hat{u}_{3}^{1}\left(x_{3}\right) \mathbf{e}_{3}$ and a rotation with the components $\partial_{3} \hat{u}_{1}^{0}\left(x_{3}\right), \partial_{3} \hat{u}_{2}^{0}\left(x_{3}\right)$, $\varphi^{1}\left(x_{3}\right)$.

\subsubsection{Microscopic Problem at $\varepsilon^{0}$ Order: $P_{2 D}^{0}$}

This time the data of the problem is $\mathbf{u}^{1}$, since $\boldsymbol{\sigma}^{0}=\mathbf{0}$, again the problem is well posed. According to the expression of $\mathbf{u}^{1}$ in Eq. (44) and due to the linearity of the problem, as presented in [4] and [6] the displacement at the second order $\mathbf{u}^{2}\left(y_{\beta}, x_{3}\right)$ has the following form:

$$
\left\{\begin{array}{c}
\mathbf{u}^{2}\left(y_{\beta}, x_{3}\right)=\dot{\mathbf{u}}^{2}\left(y_{\beta}, x_{3}\right)+\chi^{1 E}\left(y_{\beta}\right) \partial_{3} \hat{u}_{3}^{1}\left(x_{3}\right)+\chi^{1 C_{\alpha}}\left(y_{\beta}\right) \partial_{33} \hat{u}_{\alpha}^{0}\left(x_{3}\right) \\
+\chi^{1 T}\left(y_{\beta}\right) \partial_{3} \varphi^{1}\left(x_{3}\right), \\
\text { with } \hat{\mathbf{u}}^{2}\left(y_{\beta}, x_{3}\right)=\hat{u}_{i}^{2}\left(x_{3}\right) \mathbf{e}_{\mathbf{i}}+\varphi^{2}\left(x_{3}\right)\left[y_{1} \mathbf{e}_{2}-y_{2} \mathbf{e}_{1}\right]-y_{\alpha} \cdot \partial_{3} \hat{u}_{\alpha}^{1}\left(x_{3}\right) \mathbf{e}_{3},
\end{array}\right.
$$

with $\chi^{1 E}\left(y_{\beta}\right), \chi^{1 C_{\alpha}}\left(y_{\beta}\right)$ and $\chi^{1 T}\left(y_{\beta}\right)$ (vectors with respect to the variable $\mathbf{y}$ ) the displacement field corresponding respectively to the first-orde macroscopic strain composed of an extension $(E)$, two curvatures $(C)$ and a torsion $(T)$, associated respectively to $\partial_{3} \hat{u}_{3}^{1}\left(x_{3}\right)$, $\partial_{33} \hat{u}_{\alpha}^{0}\left(x_{3}\right)$ and $\partial_{3} \varphi^{1}\left(x_{3}\right)$.

In order to make the notation more concise, a four components vector $\mathbf{e}^{1}\left(x_{3}\right)$ and a $3 \times 4$ matrix $\chi^{1}\left(y_{\beta}\right)$ are define here:

$$
\mathbf{u}^{2}\left(y_{\beta}, x_{3}\right)=\mathbf{u}^{2}\left(y_{\beta}, x_{3}\right)+\chi^{1}\left(y_{\beta}\right) \dot{e}^{1}\left(x_{3}\right),
$$

with

$$
\left\{\begin{array}{l}
\chi^{1}\left(y_{\beta}\right)=\left[\chi^{1 E}\left(y_{\beta}\right), \chi^{1 C_{1}}\left(y_{\beta}\right), \chi^{1 C_{2}}\left(y_{\beta}\right), \chi^{1 T}\left(y_{\beta}\right)\right] \\
\mathbf{e}^{1}\left(x_{3}\right)={ }^{t}\left\{\partial_{3} \hat{u}_{3}^{1}\left(x_{3}\right), \partial_{33} \hat{u}_{1}^{0}\left(x_{3}\right), \partial_{33} \hat{u}_{2}^{0}\left(x_{3}\right), \partial_{3} \varphi^{1}\left(x_{3}\right)\right\}
\end{array}\right.
$$


The frst order stress $\sigma^{1}\left(y_{\beta}, x_{3}\right)$ in Eq. $(40)_{2}$ (when $k=0$ ) is then under the form of a linear function of four macroscopic strain:

$$
\boldsymbol{\sigma}^{1}\left(y_{\beta}, x_{3}\right)=\boldsymbol{\tau}^{1 E}\left(y_{\beta}\right) \partial_{3} \hat{u}_{3}^{1}\left(x_{3}\right)+\boldsymbol{\tau}^{1 C_{\alpha}}\left(y_{\beta}\right) \partial_{33} \hat{u}_{\alpha}^{0}\left(x_{3}\right)+\boldsymbol{\tau}^{1 T}\left(y_{\beta}\right) \partial_{3} \varphi^{1}\left(x_{3}\right),
$$

which can be expressed as:

$$
\boldsymbol{\sigma}^{1}\left(y_{\beta}, x_{3}\right)=\boldsymbol{\tau}^{1}\left(y_{\beta}\right) \dot{e}^{1}\left(x_{3}\right),
$$

with $\boldsymbol{\tau}^{1}\left(y_{\beta}\right)$ corresponding to the following four elementary stress solutions:

$$
\boldsymbol{\tau}^{1}\left(y_{\beta}\right)=\left[\boldsymbol{\tau}^{1 E}\left(y_{\beta}\right), \boldsymbol{\tau}^{1 C_{1}}\left(y_{\beta}\right), \boldsymbol{\tau}^{1 C_{2}}\left(y_{\beta}\right), \boldsymbol{\tau}^{1 T}\left(y_{\beta}\right)\right] .
$$

Analytical solutions for the $P_{2 D}^{0}$ problem are available for the case of an homogeneous cross-section made of isotropic material, [21]:

$$
\left\{\begin{array}{l}
\chi^{1 E}\left(y_{1}, y_{2}\right)={ }^{t}\left\{-v y_{1} ;-v y_{2} ; 0\right\} \\
\chi^{1 C_{\alpha}}\left(y_{1}, y_{2}\right)={ }^{t}\left\{v \Phi_{1 \alpha} ; v \Phi_{2 \alpha} ; 0\right\} \\
\chi^{1 T}\left(y_{1}, y_{2}\right)={ }^{t}\left\{0 ; 0 ; \varpi\left(y_{1}, y_{2}\right)\right\}
\end{array}\right.
$$

with:

$$
\left[\Phi_{\alpha \beta}\right] \equiv\left[\begin{array}{cc}
\frac{1}{2}\left(y_{1}^{2}-y_{2}^{2}\right) & y_{1} y_{2} \\
y_{1} y_{2} & \frac{1}{2}\left(y_{2}^{2}-y_{1}^{2}\right)
\end{array}\right],
$$

and $\varpi\left(y_{1}, y_{2}\right)$ the unique solution of the following torsion problem posed on the scaled cross-section $S$ of the beam structure:

$$
\begin{cases}\partial_{\alpha \alpha} \varpi=0 & \text { in } S \\ \partial_{n} \varpi=y_{2} n_{1}-y_{1} n_{2} & \text { on } \partial S \\ \int_{S} \varpi d S=0 & \end{cases}
$$

In [21] the local stress $\boldsymbol{\tau}^{1}\left(y_{\beta}\right)$ is given as:

$$
\begin{cases}\boldsymbol{\tau}^{1 E}\left(y_{1}, y_{2}\right) & =E \mathbf{e}_{3} \otimes \mathbf{e}_{3} \\ \boldsymbol{\tau}^{1 C_{1}}\left(y_{1}, y_{2}\right) & =-E y_{1} \mathbf{e}_{3} \otimes \mathbf{e}_{3} \\ \boldsymbol{\tau}^{1 C_{2}}\left(y_{1}, y_{2}\right) & =-E y_{2} \mathbf{e}_{3} \otimes \mathbf{e}_{3} \\ \boldsymbol{\tau}^{1 T}\left(y_{1}, y_{2}\right) & =G\left(\partial_{2} \Psi\left(\mathbf{e}_{1} \otimes \mathbf{e}_{3}+\mathbf{e}_{3} \otimes \mathbf{e}_{1}\right)-\partial_{1} \Psi\left(\mathbf{e}_{2} \otimes \mathbf{e}_{3}+\mathbf{e}_{3} \otimes \mathbf{e}_{2}\right)\right)\end{cases}
$$

with $G$ the shear modulus, $\Psi$ corresponds to the Prandtl function given by:

$$
\left\{\begin{array}{l}
\partial_{2} \Psi=\partial_{1} \varpi-y_{2} \\
\partial_{1} \Psi=-\partial_{2} \varpi-y_{1}
\end{array}\right.
$$

\subsubsection{Microscopic Problem at $\varepsilon^{1}$ Order: $P_{2 D}^{1}$}

At this order, the term $-\mathbf{d i v}_{x_{3}} \boldsymbol{\sigma}^{1}$ is non zero. $P_{2 D}^{1}$ problem admits a solution if and only if the load $-\mathbf{d i v}_{x_{3}} \boldsymbol{\sigma}^{1}$ satisfie the existence condition Eq. (42). The compatibility equation Eq. (42) will be used to derive the firs order macroscopic problem, see Sect. 4.2.1. 
According to the expression of $\mathbf{u}^{2}$ in Eq. (46), the displacement fiel $\mathbf{u}^{3}$ is now obtained as [4]:

$$
\left\{\begin{array}{l}
\mathbf{u}^{3}=\dot{\mathbf{u}}^{3}\left(y_{\beta}, x_{3}\right)+\chi^{1}\left(y_{\beta}\right) \dot{\mathbf{e}}^{2}\left(x_{3}\right)+\chi^{2}\left(y_{\beta}\right) \partial_{3} \dot{\mathbf{e}}^{1}\left(x_{3}\right), \\
\text { with }\left\{\begin{array}{l}
\dot{\mathbf{u}}^{3}\left(y_{\beta}, x_{3}\right)=\hat{u}_{i}^{3}\left(x_{3}\right) \mathbf{e}_{\mathbf{i}}+\varphi^{3}\left(x_{3}\right)\left[y_{1} \mathbf{e}_{2}-y_{2} \mathbf{e}_{1}\right]-y_{\alpha} \partial_{3} \hat{u}_{\alpha}^{2}\left(x_{3}\right) \mathbf{e}_{3}, \\
\chi^{2}\left(y_{\beta}\right)=\left[\chi^{2 E}\left(y_{\beta}\right), \chi^{2 C_{1}}\left(y_{\beta}\right), \chi^{2 C_{2}}\left(y_{\beta}\right), \chi^{2 T}\left(y_{\beta}\right)\right]
\end{array}\right.
\end{array}\right.
$$

with the second order macroscopic strain:

$$
\mathbf{e}^{2}\left(x_{3}\right)={ }^{t}\left\{\partial_{3} \hat{u}_{3}^{2}\left(x_{3}\right), \partial_{33} \hat{u}_{1}^{1}\left(x_{3}\right), \partial_{33} \hat{u}_{2}^{1}\left(x_{3}\right), \partial_{3} \varphi^{2}\left(x_{3}\right)\right\},
$$

and the gradient of the firs order macroscopic strain:

$$
\partial_{3} \mathbf{e}^{1}\left(x_{3}\right)={ }^{t}\left\{\partial_{33} \hat{u}_{3}^{1}\left(x_{3}\right), \partial_{333} \hat{u}_{1}^{0}\left(x_{3}\right), \partial_{333} \hat{u}_{2}^{0}\left(x_{3}\right), \partial_{33} \varphi^{1}\left(x_{3}\right)\right\},
$$

thus $\chi^{2 E}\left(y_{\beta}\right), \chi^{2 C_{\alpha}}\left(y_{\beta}\right)$ and $\chi^{2 T}\left(y_{\beta}\right)$ are new local displacement $\mathrm{f}$ elds corresponding to the gradient of the $\mathrm{f}$ rst-order strain.

The stress fiel at second order, $\sigma^{2}$, is then obtained as:

$$
\boldsymbol{\sigma}^{2}\left(y_{\beta}, x_{3}\right)=\boldsymbol{\tau}^{1}\left(y_{\beta}\right) \dot{\mathbf{e}}^{2}\left(x_{3}\right)+\boldsymbol{\tau}^{2}\left(y_{\beta}\right) \partial_{3} \mathbf{e}^{1}\left(x_{3}\right),
$$

with

$$
\boldsymbol{\tau}^{2}\left(y_{\beta}\right)=\left[\boldsymbol{\tau}^{2 E}\left(y_{\beta}\right), \boldsymbol{\tau}^{2 C_{1}}\left(y_{\beta}\right), \boldsymbol{\tau}^{2 C_{2}}\left(y_{\beta}\right), \boldsymbol{\tau}^{2 T}\left(y_{\beta}\right)\right] .
$$

The stress fiel $\boldsymbol{\tau}^{1}\left(y_{\beta}\right)$ is already determined by solving the $P_{2 D}^{0}$ problem Eq. (51), while the four components of $\boldsymbol{\tau}^{2}\left(y_{\beta}\right)$ are four new elementary solutions of the $P_{2 D}^{1}$ problem, where the four components of $\partial_{3} \mathbf{e}^{1}\left(x_{3}\right)$ are prescribed.

Analytical solutions for the case of an homogeneous cross-section made of isotropic material are available in [21], while $\chi^{2 E}$ and $\tau^{2 T}$ are not given in this article, the general form of the solution for $\chi^{2 C_{\beta}}$ is:

$$
\chi^{2 C_{\beta}}\left(y_{1}, y_{2}\right)={ }^{t}\left\{0 ; 0 ; v \theta_{\beta}\left(y_{1}, y_{2}\right)+(1+v) \eta_{\beta}\left(y_{1}, y_{2}\right)\right\}
$$

where $\theta_{\beta}\left(y_{1}, y_{2}\right)$ and $\eta_{\beta}\left(y_{1}, y_{2}\right)$ are respectively the unique solutions of the following problems:

$$
\left\{\begin{array} { l l } 
{ - \partial _ { \alpha \alpha } \theta _ { \beta } = 2 y _ { \beta } } & { \text { in } S , } \\
{ \partial _ { n } \theta _ { \beta } = - \Phi _ { \alpha \beta } n _ { \alpha } } & { \text { on } \partial S , } \\
{ \int _ { S } \theta _ { \beta } d S = 0 , } & { }
\end{array} \left\{\begin{array}{ll}
-\partial_{\alpha \alpha} \eta_{\beta}=-2 y_{\beta} & \text { in } S, \\
\partial_{n} \eta_{\beta}=0 & \text { on } \partial S, \\
\int_{S} \eta_{\beta} d S=0, &
\end{array}\right.\right.
$$

and

$$
\left\{\begin{aligned}
\boldsymbol{\tau}^{2 C_{1}}\left(y_{1}, y_{2}\right)= & G\left[v \Phi_{11}+v \partial_{1} \theta_{1}+(1+v) \partial_{1} \eta_{1}\right]\left(\mathbf{e}_{\mathbf{1}} \otimes \mathbf{e}_{3}+\mathbf{e}_{3} \otimes \mathbf{e}_{\mathbf{1}}\right) \\
& +G\left[v \Phi_{21}+v \partial_{2} \theta_{1}+(1+v) \partial_{2} \eta_{1}\right]\left(\mathbf{e}_{\mathbf{2}} \otimes \mathbf{e}_{\mathbf{3}}+\mathbf{e}_{\mathbf{3}} \otimes \mathbf{e}_{2}\right), \\
\boldsymbol{\tau}^{2 C_{2}}\left(y_{1}, y_{2}\right)= & G\left[v \Phi_{12}+v \partial_{1} \theta_{2}+(1+v) \partial_{1} \eta_{2}\right]\left(\mathbf{e}_{\mathbf{1}} \otimes \mathbf{e}_{3}+\mathbf{e}_{\mathbf{3}} \otimes \mathbf{e}_{1}\right) \\
& +G\left[v \Phi_{22}+v \partial_{2} \theta_{2}+(1+v) \partial_{2} \eta_{2}\right]\left(\mathbf{e}_{\mathbf{2}} \otimes \mathbf{e}_{\mathbf{3}}+\mathbf{e}_{\mathbf{3}} \otimes \mathbf{e}_{2}\right) .
\end{aligned}\right.
$$


Analytical solutions for the functions $\theta_{\alpha}\left(y_{1}, y_{2}\right)$ and $\eta_{\alpha}\left(y_{1}, y_{2}\right)$ are available for a circular cross-section of radius $R$ [21]:

$$
\left\{\begin{array}{l}
\theta_{\alpha}=-\frac{1}{4} y_{\alpha}\left(y_{1}^{2}+y_{2}^{2}-\varepsilon^{-2} R^{2}\right), \\
\eta_{\alpha}=\frac{1}{4} y_{\alpha}\left(y_{1}^{2}+y_{2}^{2}-3 \varepsilon^{-2} R^{2}\right) .
\end{array}\right.
$$

\subsubsection{Asymptotic Expansion Solution Fields of the Initial 3D Problem}

Solving the 2D microscopic problems recursively leads to the general form of higher order microscopic problems, which are presented in [21] and [6], therefore the following expression for the asymptotic expansion solution are determined [21]:

$$
\begin{aligned}
\mathbf{u}^{\varepsilon}\left(y_{\beta}, x_{3}\right)= & \hat{u}_{\alpha}^{0}\left(x_{3}\right) \mathbf{e}_{\alpha} \\
& +\varepsilon^{1}\left[\dot{\mathbf{u}}^{1}\left(y_{\beta}, x_{3}\right)\right] \\
& +\varepsilon^{2}\left[\dot{\mathbf{u}}^{2}\left(y_{\beta}, x_{3}\right)+\chi^{1}\left(y_{\beta}\right) \cdot \mathbf{e}^{1}\left(x_{3}\right)\right] \\
& +\varepsilon^{3}\left[\dot{\mathbf{u}}^{3}\left(y_{\beta}, x_{3}\right)+\chi^{1}\left(y_{\beta}\right) \cdot \mathbf{e}^{2}\left(x_{3}\right)+\chi^{2}\left(y_{\beta}\right) \cdot \partial_{3} \dot{\mathbf{e}}^{1}\left(x_{3}\right)\right] \\
& +\varepsilon^{4}\left[\dot{\mathbf{u}}^{4}\left(y_{\beta}, x_{3}\right)+\chi^{1}\left(y_{\beta}\right) \cdot \dot{\mathbf{e}}^{3}\left(x_{3}\right)+\chi^{2}\left(y_{\beta}\right) \cdot \partial_{3} \dot{\mathbf{e}}^{2}\left(x_{3}\right)+\chi^{3}\left(y_{\beta}\right) \cdot \partial_{33} \mathbf{e}^{1}\left(x_{3}\right)\right] \\
& +\cdots,
\end{aligned}
$$

and for the stress fiel $\boldsymbol{\sigma}^{\varepsilon}\left(y_{\beta}, x_{3}\right)$ :

$$
\begin{aligned}
\boldsymbol{\sigma}^{\varepsilon}\left(y_{\beta}, x_{3}\right)= & \varepsilon^{1}\left[\boldsymbol{\tau}^{1}\left(y_{\beta}\right) \cdot \mathbf{e}^{1}\left(x_{3}\right)\right] \\
& +\varepsilon^{2}\left[\boldsymbol{\tau}^{1}\left(y_{\beta}\right) \cdot \dot{\mathbf{e}}^{-2}\left(x_{3}\right)+\boldsymbol{\tau}^{2}\left(y_{\beta}\right) \cdot \partial_{3} \dot{\mathbf{e}}^{1}\left(x_{3}\right)\right] \\
& +\varepsilon^{3}\left[\boldsymbol{\tau}^{1}\left(y_{\beta}\right) \cdot \dot{\mathbf{e}}^{3}\left(x_{3}\right)+\boldsymbol{\tau}^{2}\left(y_{\beta}\right) \cdot \partial_{3} \dot{\mathbf{e}}^{2}\left(x_{3}\right)+\boldsymbol{\tau}^{3}\left(y_{\beta}\right) \cdot \partial_{33} \mathbf{e}^{1}\left(x_{3}\right)\right] \\
& +\cdots,
\end{aligned}
$$

in Eqs. (66) and (67) solutions are constituted of two parts: the microscopic part $\left(\chi^{p}\left(y_{\beta}\right)\right.$, $\left.\boldsymbol{\tau}^{p}\left(y_{\beta}\right)\right)$ and the macroscopic part $\left(\dot{\mathbf{u}}^{p}\left(y_{\beta}, x_{3}\right)\right.$, $\mathbf{e}^{p}\left(x_{3}\right)$, can be define in a recursive manner from: Eqs. (44), (46), (48) and (58)). Some analytical solutions relative to the frst two microscopic problems $\left(P_{2 D}^{0}\right.$ and $\left.P_{2 D}^{1}\right)$ have been provided. It is shown in the following that this is sufficien for the homogeneous case.

\subsection{Macroscopic Problems and Solutions}

The derivation of the macroscopic problems is made in [20] and [21], but due to boundary conditions at terminal sections considered in these references, there are no analytical solution available for the 3D elastic problem, or for the macroscopic problems. In the following, it is shown that for the case of homogeneous isotropic beams and Saint-Venant problems, the asymptotic expansion has a f nite number of terms and both elastic 3D problem, microscopic and macroscopic problems have analytical solution. As mentioned in Sect. 4.1, and according to [20,21] and [6], the macroscopic problems $\left(P_{h o m}^{k}\right)$ are formulated with the help of the compatibility condition Eq. (42). By expressing Eq. (42) for the 2D-problems $P_{2 D}^{k}$ and $P_{2 D}^{k+1}$, equilibrium equations related to the macroscopic problems are obtained. 


\subsubsection{At $\varepsilon^{1}$ Order: $P_{\text {hom }}^{1}$}

Equilibrium Equations As mentioned in Sect. 4.1.3, the equilibrium equations of $P_{h o m}^{1}$ are obtained following the compatibility condition Eq. (42) for the existence of a solution for the $P_{2 D}^{1}$ problem, more details can be found in [6]:

$$
\left\{\begin{array}{l}
\partial_{3} N^{1}\left(x_{3}\right)=0 \\
\partial_{3} T_{\alpha}^{2}\left(x_{3}\right)=0 \\
\partial_{3} M_{3}^{1}\left(x_{3}\right)=0 \\
-T_{\alpha}^{2}\left(x_{3}\right)+\partial_{3} M_{\alpha}^{1}\left(x_{3}\right)=0
\end{array}\right.
$$

with:

$$
\left\{\begin{array}{l}
N^{1}\left(x_{3}\right)=\left\langle\sigma_{33}^{1}\left(y_{\beta}, x_{3}\right)\right\rangle, \\
T_{\alpha}^{2}\left(x_{3}\right)=\left\langle\sigma_{\alpha 3}^{2}\left(y_{\beta}, x_{3}\right)\right\rangle, \\
M_{\alpha}^{1}\left(x_{3}\right)=\left\langle-y_{\alpha} \sigma_{33}^{1}\left(y_{\beta}, x_{3}\right)\right\rangle, \\
M_{3}^{1}\left(x_{3}\right)=\left\langle\left(-y_{2} \sigma_{13}^{1}\left(y_{\beta}, x_{3}\right)+y_{1} \sigma_{23}^{1}\left(y_{\beta}, x_{3}\right)\right)\right\rangle,
\end{array}\right.
$$

which is the same as in Eq. (36) with $k=1$ and $k=2$, denoting the normal force, shear forces, bending moments and torsion moment at a certain order $\varepsilon^{p}$.

Constitutive Equations Considering the form of the stresses derived from the solutions of the 0th order microscopic problems Eq. (49), the macroscopic behavior can be written as:

$$
\left\{\begin{array}{l}
N^{1}\left(x_{3}\right) \\
M_{1}^{1}\left(x_{3}\right) \\
M_{2}^{1}\left(x_{3}\right) \\
M_{3}^{1}\left(x_{3}\right)
\end{array}\right\}=\mathcal{A}^{\text {hom } 1} \cdot\left\{\begin{array}{c}
\partial_{3} \hat{u}_{3}^{1}\left(x_{3}\right) \\
\partial_{33} \hat{u}_{1}^{0}\left(x_{3}\right) \\
\partial_{33} \hat{u}_{2}^{0}\left(x_{3}\right) \\
\partial_{3} \varphi^{1}\left(x_{3}\right)
\end{array}\right\}
$$

or:

$$
\dot{\sigma}^{1}\left(x_{3}\right)=\mathcal{A}^{\text {hom } 1} \cdot \mathbf{e}^{1}\left(x_{3}\right),
$$

with:

$$
\dot{\boldsymbol{\sigma}}^{1}\left(x_{3}\right)={ }^{t}\left\{N^{1}\left(x_{3}\right), M_{1}^{1}\left(x_{3}\right), M_{2}^{1}\left(x_{3}\right), M_{3}^{1}\left(x_{3}\right)\right\},
$$

and the components of the matrix $\mathcal{A}^{\text {hom } 1}$ given by:

$$
\begin{array}{ll}
\mathcal{A}_{1 \mathcal{L}}^{\text {hom } 1}=\left\langle\tau_{33}^{1 \mathcal{L}}\left(y_{\beta}\right)\right\rangle, & \mathcal{A}_{2 \mathcal{L}}^{\text {hom } 1}=\left\langle-y_{1} \tau_{33}^{1 \mathcal{L}}\left(y_{\beta}\right)\right\rangle, \\
\mathcal{A}_{3 \mathcal{L}}^{\text {hom } 1}=\left\langle-y_{2} \tau_{33}^{1 \mathcal{L}}\left(y_{\beta}\right)\right\rangle, \quad \mathcal{A}_{4 \mathcal{L}}^{\text {hom } 1}=\left\langle-y_{2} \tau_{13}^{1 \mathcal{L}}\left(y_{\beta}\right)+y_{1} \tau_{23}^{1 \mathcal{L}}\left(y_{\beta}\right)\right\rangle,
\end{array}
$$

where $\tau_{i j}^{1 \mathcal{L}}(\mathbf{y})(\mathcal{L} \in\{1,2,3,4\})$ is define by: $\tau_{i j}^{11}=\tau_{i j}^{1 E}, \tau_{i j}^{12}=\tau_{i j}^{1 C_{1}}, \tau_{i j}^{13}=\tau_{i j}^{1 C_{2}}$ and $\tau_{i j}^{14}=\tau_{i j}^{1 T}$, solutions of the $\varepsilon^{0}$ order microscopic problem $P_{2 D}^{0}$.

Remark In Eq. (70) no expressions for the shear force appear. However, as mentioned in Sect. 4.1.3, a shear problem can be solved considering the gradient of the macroscopic strain given at higher order (see Sect. 5). 
Analytical solutions in Eq. (72) are available for the case of a homogeneous isotropic cross-section with $\tau^{1 E}, \tau^{1 C_{\alpha}}, \tau^{1 T}$ given in Eq. (55) [4]:

$$
\mathcal{A}^{\text {hom } 1} \equiv\left[\begin{array}{cccc}
E|S| & 0 & 0 & 0 \\
0 & E I_{1} & 0 & 0 \\
0 & 0 & E I_{2} & 0 \\
0 & 0 & 0 & G J
\end{array}\right]
$$

with $|S|$ the area of the scaled cross-section: $|S|=\int_{S} d y_{1} y_{2}$, and $I_{\alpha}$ the two second moments of area:

$$
I_{\alpha}=\int_{S} y_{\alpha}^{2} d y_{1} y_{2}
$$

and so f nally, one has from Eq. (13):

$$
I_{\alpha}^{\varepsilon}=\varepsilon^{4} I_{\alpha}
$$

$J$ is the torsion constant for the scaled cross-section:

$$
J=-\int_{S} y_{\alpha} \partial_{\alpha} \Psi d y_{1} y_{2}=I_{1}+I_{2}-\int_{S}\left[\left(\partial_{1} \varpi\right)^{2}+\left(\partial_{2} \varpi\right)^{2}\right] d y_{1} y_{2} .
$$

Then according to Eq. (17):

$$
J^{\varepsilon}=\varepsilon^{4} J
$$

For a circular cross-section the torsion warping function $\varpi$ is zero. Then:

$$
J=I_{1}+I_{2}
$$

Boundary Conditions To solve the macroscopic 1D-problem $P_{\text {hom }}^{1}$, it still remains to complete the governing equations Eqs. (68) and (70) with the boundary conditions at the two ends of the structure. According to Eq. (39) (when $k=1$ or 2), the boundary conditions at this order for the two ends $S_{0}$ and $S_{L}$ are:

$$
\left\{\begin{array} { l } 
{ N ^ { 1 } ( 0 ) = N _ { L } ^ { \prime } , } \\
{ T _ { \alpha } ^ { 2 } ( 0 ) = T _ { \alpha _ { L } } ^ { \prime } , } \\
{ M _ { \alpha } ^ { 1 } ( 0 ) = ( M _ { \alpha _ { L } } ^ { \prime } + T _ { \alpha _ { L } } ^ { \prime } L ) , } \\
{ M _ { 3 } ^ { 1 } ( 0 ) = M _ { 3 _ { L } } ^ { \prime } , }
\end{array} \text { and } \left\{\begin{array}{l}
N^{1}(L)=N_{L}^{\prime}, \\
T_{\alpha}^{2}(L)=T_{\alpha_{L}}^{\prime}, \\
M_{\alpha}^{1}(L)=M_{\alpha_{L}}^{\prime}, \\
M_{3}^{1}(L)=M_{3_{L}}^{\prime} .
\end{array}\right.\right.
$$

The general form of the macroscopic solution becomes:

$$
\left\{\begin{array}{l}
N^{1}\left(x_{3}\right)=N_{L}^{\prime} \\
T_{\alpha}^{2}\left(x_{3}\right)=T_{\alpha_{L}}^{\prime} \\
M_{\alpha}^{1}\left(x_{3}\right)=M_{\alpha_{L}}^{\prime}+T_{\alpha_{L}}^{\prime}\left(L-x_{3}\right) \\
M_{3}^{1}\left(x_{3}\right)=M_{3_{L}}^{\prime}
\end{array}\right.
$$

The $P_{\text {hom }}^{1}$ problem generalizes and justifie the Euler-Bernoulli-Navier's beam model. Its mathematical justification using convergence results, can be found in [15]. Finally it should be mentioned that due to the form of the loadings in Eq. (80), é $^{1}\left(x_{3}\right)$ is linear. 


\subsubsection{At Higher Order: $P_{\text {hom }}^{k}$}

Equilibrium Equations By the same method applied in the preceding section, the formulation of the higher-order problems are obtained. The equilibrium equations are $[4,6]$ :

$$
\left\{\begin{array}{l}
\partial_{3} N^{k}\left(x_{3}\right)=0, \\
\partial_{3} T_{\alpha}^{k+1}\left(x_{3}\right)=0, \\
\partial_{3} M_{3}^{k}\left(x_{3}\right)=0, \\
-T_{\alpha}^{k+1}\left(x_{3}\right)+\partial_{3} M_{\alpha}^{k}\left(x_{3}\right)=0,
\end{array} \quad k \geq 2,\right.
$$

with $N^{k}, T_{\alpha}^{k+1}, M_{\alpha}^{k}$ and $M_{3}^{k}$ define in Eq. (36).

Constitutive Equations According to the solutions $\sigma^{k}$ and $\sigma^{k+1}$ of the $P_{2 D}^{k}$ and $P_{2 D}^{k+1}$ microscopic problems, the macroscopic behavior is of the form [6]:

$$
\begin{aligned}
\dot{\boldsymbol{\sigma}}^{k}\left(x_{3}\right)= & \mathcal{A}^{h o m 1} \cdot \dot{\mathbf{e}}^{k}\left(x_{3}\right)+\mathcal{A}^{h o m 2} . \partial_{3} \dot{\mathbf{e}}^{k-1}\left(x_{3}\right)+\mathcal{A}^{h o m 3} . \partial_{33} \dot{\mathbf{e}}^{k-2}\left(x_{3}\right)+\cdots \\
& +\mathcal{A}^{h o m k} . \partial_{3}^{k-1} \dot{\mathbf{e}}^{k-1}\left(x_{3}\right),
\end{aligned}
$$

where $\hat{\boldsymbol{\sigma}}^{k}\left(x_{3}\right)={ }^{t}\left\{N^{k}, \boldsymbol{M}_{1}^{k}, M_{2}^{k}, M_{3}^{k}\right\}$, and $\hat{\mathbf{e}}^{k}\left(x_{3}\right)={ }^{t}\left\{\partial_{3} \hat{u}_{3}^{k}, \partial_{33} \hat{u}_{1}^{k-1}, \partial_{33} \hat{u}_{2}^{k-1}, \partial_{3} \varphi^{k}\right\}$.

- The 2nd-order problem: $P_{h o m}^{2}$

In Eq. (82), when $k=2$, we have:

$$
\dot{\boldsymbol{\sigma}}^{2}\left(x_{3}\right)=\mathcal{A}^{\text {hom } 1} \cdot \dot{\mathbf{e}}^{2}\left(x_{3}\right)+\mathcal{A}^{\text {hom } 2} \cdot \partial_{3} \mathbf{e}^{1}\left(x_{3}\right) .
$$

Contrary to the firs order effective stiffness matrix $\mathcal{A}^{\text {hom } 1}$, it is proved in [3] and [5] that $\mathcal{A}^{\text {hom } 2}$ is anti-symmetric and equals to zero owning to certain symmetry properties of the cross-section [4]; then we have:

$$
\dot{\boldsymbol{\sigma}}^{2}\left(x_{3}\right)=\mathcal{A}^{\text {hom } 1} \cdot \mathbf{e}^{2}\left(x_{3}\right) .
$$

Boundary Conditions To complete the formulation of the higher order problems, the boundary conditions at the two end sections must be defined According to Eq. (39) (when $k=2, k=3$ ), the boundary conditions at this order for $x_{3}=0$ and $x_{3}=L$ are:

$$
\left\{\begin{array} { l } 
{ N ^ { 2 } ( 0 ) = 0 , } \\
{ T _ { \alpha } ^ { 3 } ( 0 ) = 0 , } \\
{ M _ { \alpha } ^ { 2 } ( 0 ) = 0 , } \\
{ M _ { 3 } ^ { 2 } ( 0 ) = - y _ { 2 C } T _ { 1 _ { L } } ^ { \prime } + y _ { 1 C } T _ { 2 _ { L } } ^ { \prime } , }
\end{array} \text { and } \quad \left\{\begin{array}{l}
N^{2}(L)=0, \\
T_{\alpha}^{3}(L)=0, \\
M_{\alpha}^{2}(L)=0, \\
M_{3}^{2}(L)=-y_{2 C} T_{1_{L}}^{\prime}+y_{1 C} T_{2_{L}}^{\prime} .
\end{array}\right.\right.
$$

The general form of the macroscopic solution at this order is:

$$
\left\{\begin{array}{l}
N^{2}\left(x_{3}\right)=0 \\
T_{\alpha}^{3}\left(x_{3}\right)=0 \\
M_{\alpha}^{2}\left(x_{3}\right)=0 \\
M_{3}^{2}\left(x_{3}\right)=-y_{2 C} T_{1_{L}}^{\prime}+y_{1 C} T_{2_{L}}^{\prime}
\end{array}\right.
$$


It is easy to $\mathrm{f}$ nd that the only non-zero component for the loadings is the higher order torsion:

$$
\dot{\boldsymbol{\sigma}}^{2}\left(x_{3}\right)={ }^{t}\left\{0,0,0,-y_{2 C} T_{1_{L}}^{\prime}+y_{1 C} T_{2_{L}}^{\prime}\right\}
$$

For the homogeneous case one has from Eqs. (70) and (73)

$$
\mathbf{e}^{2}\left(x_{3}\right)={ }^{t}\left\{0,0,0, \partial_{3} \varphi^{2}\right\},
$$

with $\partial_{3} \varphi^{2}=\left(-y_{2 C} T_{1_{L}}^{\prime}+y_{1 C} T_{2_{L}}^{\prime}\right) /(G J)$. Thus, for a homogeneous beam with loadings applied at its end sections $\mathbf{e}^{2}\left(x_{3}\right)$ is a constant and $\partial_{3} \mathbf{e}^{2}\left(x_{3}\right)$ is zero.

- The 3rd-order problem: $P_{\text {hom }}^{3}$

In Eq. (82), when $k=3$, we have:

$$
\dot{\boldsymbol{\sigma}}^{3}\left(x_{3}\right)=\mathcal{A}^{\text {hom } 1} \cdot \dot{\mathbf{e}}^{3}\left(x_{3}\right)+\mathcal{A}^{\text {hom } 3} \cdot \partial_{33} \dot{e}^{1}\left(x_{3}\right) .
$$

Due to the form of $\mathbf{e}^{1}\left(x_{3}\right)$, we have $\partial_{33} \mathbf{e}^{1}\left(x_{3}\right)=0$ and:

$$
\boldsymbol{\sigma}^{3}\left(x_{3}\right)=\mathcal{A}^{h o m 1} \cdot \mathbf{e}^{3}\left(x_{3}\right) .
$$

According to Eq. (39) $)_{3}$, the boundary conditions at this order for $x_{3}=0$ and $x_{3}=L$ are:

$$
\left\{\begin{array} { l } 
{ N ^ { 3 } ( 0 ) = 0 , } \\
{ T _ { \alpha } ^ { 4 } ( 0 ) = 0 , } \\
{ M _ { \alpha } ^ { 3 } ( 0 ) = 0 , } \\
{ M _ { 3 } ^ { 3 } ( 0 ) = 0 , }
\end{array} \quad \text { and } \quad \left\{\begin{array}{l}
N^{3}(L)=0, \\
T_{\alpha}^{4}(L)=0, \\
M_{\alpha}^{3}(L)=0, \\
M_{3}^{3}(L)=0 .
\end{array}\right.\right.
$$

The general form of the macroscopic solution at this order is:

$$
\left\{\begin{array}{l}
N^{3}\left(x_{3}\right)=0 \\
T_{\alpha}^{4}\left(x_{3}\right)=0 \\
M_{\alpha}^{3}\left(x_{3}\right)=0 \\
M_{3}^{3}\left(x_{3}\right)=0
\end{array}\right.
$$

and for $\mathcal{A}^{\text {hom } 1} \neq 0$, we have:

$$
\dot{\boldsymbol{\sigma}}^{3}\left(x_{3}\right)=\mathbf{e}^{3}\left(x_{3}\right)=\mathbf{0} .
$$

Recursively, it can be deduced that: for a beam with loadings applied at its ends, é $^{1}\left(x_{3}\right)$ is linear, $\mathbf{e}^{2}\left(x_{3}\right)$ is a constant, and $\mathbf{e}^{k}\left(x_{3}\right)=\mathbf{0}(k \geq 3)$. The second gradient of the strain $\partial_{33} \dot{e}^{k}\left(x_{3}\right)=\mathbf{0}$ at each order. The stress fiel in Eq. (67) reduces to:

$$
\boldsymbol{\sigma}^{\varepsilon}\left(y_{\beta}, x_{3}\right)=\varepsilon^{1}\left[\boldsymbol{\tau}^{1}\left(y_{\beta}\right) \cdot \mathbf{e}^{1}\left(x_{3}\right)\right]+\varepsilon^{2}\left[\boldsymbol{\tau}^{1}\left(y_{\beta}\right) \cdot \dot{\mathbf{e}}^{2}\left(x_{3}\right)+\boldsymbol{\tau}^{2}\left(y_{\beta}\right) \cdot \partial_{3} \mathbf{e}^{1}\left(x_{3}\right)\right],
$$

or in other words the asymptotic expansion of the stress fiel stops at the $\varepsilon^{2}$ order (it's interesting to notice that the Saint-Venant problems lead to an asymptotic expansion with a finit (and small) number of terms).

The previous results are of course valid for the case when the only applied loads are at the ends of the beam. For a beam with uniformly distributed transverse loads along its length, $T_{\alpha_{L}}^{\prime}$ is linear and then solutions of one order higher are needed. 


\section{Asymptotic Expansions vs Saint-Venant Solutions}

In Sect. 4 analytical solutions for both microscopic and macroscopic problems of the asymptotic expansion method were found with the asymptotic expansion of the stress fiel given in Eq. (94). They are then used in this section for solving the Saint-Venant problems.

\subsection{Pure Bending}

For the case of pure bending (Fig. 2), only bending moments $M_{1_{L}}^{\prime}$ (equal to $\varepsilon^{-4} M_{1_{L}}^{\varepsilon}$ ) are applied on both scaled end cross-sections of the beam. Therefore, according to Eq. (39) we get:

$$
\left\{\begin{array} { l } 
{ N ^ { 1 } ( 0 ) = N ^ { 1 } ( L ) = 0 , } \\
{ M _ { 2 } ^ { 1 } ( 0 ) = M _ { 2 } ^ { 1 } ( L ) = 0 , } \\
{ M _ { 3 } ^ { 1 } ( 0 ) = M _ { 3 } ^ { 1 } ( L ) = 0 , } \\
{ T _ { \alpha } ^ { 2 } ( 0 ) = T _ { \alpha } ^ { 2 } ( L ) = 0 , }
\end{array} \text { and } \quad \left\{\begin{array}{l}
M_{1}^{1}(0)=M_{1_{L}}^{\prime}=\varepsilon^{-4} M_{1_{L}}^{\varepsilon} \\
M_{1}^{1}(L)=M_{1_{L}}^{\prime}=\varepsilon^{-4} M_{1_{L}}^{\varepsilon}
\end{array}\right.\right.
$$

The solution of the firs order macroscopic problem $P_{h o m}^{1}$ is:

$$
\begin{cases}M_{1}^{1}\left(z_{3}\right)=\varepsilon^{-4} M_{1_{L}}^{\varepsilon}, \\ M_{2}^{1}\left(z_{3}\right)=0, & N^{1}\left(x_{3}\right)=0, \\ M_{3}^{1}\left(z_{3}\right)=0, & T_{\alpha}^{2}\left(x_{3}\right)=0,\end{cases}
$$

where the macroscopic constitutive law Eqs. (70), (73) leads to:

$$
\partial_{3} \hat{u}_{3}^{1}\left(x_{3}\right)=0, \quad \partial_{3} \varphi^{1}\left(x_{3}\right)=0, \quad \partial_{33} \hat{u}_{2}^{0}\left(x_{3}\right)=0,
$$

and thus the only non-zero component of the f rst order macroscopic strain is constant:

$$
\partial_{33} \hat{u}_{1}^{0}\left(x_{3}\right)=\frac{M_{1}^{1}\left(x_{3}\right)}{E I_{1}},
$$

which, with Eqs. (26), (55), (75) and (96) 2 , lead Eq. (49) to:

$$
\boldsymbol{\sigma}^{\varepsilon}\left(y_{\beta}, x_{3}\right)=\varepsilon\left[-y_{1} E \frac{M_{1}^{1}\left(z_{3}\right)}{E I_{1}} \mathbf{e}_{3} \otimes \mathbf{e}_{3}\right]=-x_{1} \frac{M_{1_{L}}^{\varepsilon}}{I_{1}^{\varepsilon}} \mathbf{e}_{3} \otimes \mathbf{e}_{3} .
$$

This coincides with the Saint-Venant solution in Eq. (18). It is also observed that the normal stress due to a bending moment appears at the firs order of the asymptotic expansion method.

\subsection{Simple Bending}

\subsubsection{Simple Bending Problem Without Torsion}

We consider a beam loaded on its two ends with a self-balanced shear force $\varepsilon^{-4} T_{1_{L}}^{\varepsilon}$ and a bending moment $\varepsilon^{-4} M_{1_{L}}^{\varepsilon}=\varepsilon^{-4} T_{1_{L}}^{\varepsilon} . L$ on $S_{0}^{\varepsilon}$, see Fig. 3. The shear center is assumed 
on the beam axis and so no torsional effects arise. The only non-zero components of the macroscopic solution are:

$$
\left\{\begin{array}{l}
T_{1}^{2}\left(x_{3}\right)=\varepsilon^{-4} T_{1_{L}}^{\varepsilon}, \\
M_{1}^{1}\left(x_{3}\right)=\varepsilon^{-4} T_{1_{L}}^{\varepsilon}\left(L-x_{3}\right) .
\end{array}\right.
$$

The f rst order macroscopic constitutive law Eq. (70) and the analytical solution Eq. (73) lead to the four components of $\mathbf{e}^{1}\left(x_{3}\right)$ :

$$
\partial_{3} \hat{u}_{3}^{1}\left(x_{3}\right)=0, \quad \partial_{33} \hat{u}_{2}^{0}\left(x_{3}\right)=0, \quad \partial_{3} \varphi^{1}\left(x_{3}\right)=0,
$$

and

$$
\partial_{33} \hat{u}_{1}^{0}\left(x_{3}\right)=\frac{M_{1}^{1}\left(x_{3}\right)}{E I_{1}}=\frac{T_{1_{L}}^{\varepsilon}\left(L-x_{3}\right)}{E I_{1}^{\varepsilon}} .
$$

The general form of the stress fiel $\boldsymbol{\sigma}^{\varepsilon}\left(y_{\beta}, x_{3}\right)$, solution of the asymptotic expansion method, is given in Eq. (94), with $\boldsymbol{\tau}^{1}\left(y_{\beta}\right)$ known from Eq. (55). Thus, $\boldsymbol{\sigma}^{1}\left(y_{\beta}, x_{3}\right)$ is:

$$
\begin{aligned}
\boldsymbol{\sigma}^{1}\left(y_{\beta}, x_{3}\right) & =\boldsymbol{\tau}^{1}\left(y_{\beta}\right) \cdot \dot{\mathbf{e}}^{1}\left(x_{3}\right) \\
& =\left(-y_{1} E\right) \frac{T_{1_{L}}^{\varepsilon}\left(L-x_{3}\right)}{E I_{1}^{\varepsilon}} \mathbf{e}_{3} \otimes \mathbf{e}_{3} \\
& =-y_{1}\left(L-x_{3}\right) \frac{T_{1_{L}}^{\varepsilon}}{I_{1}^{\varepsilon}} \mathbf{e}_{3} \otimes \mathbf{e}_{3},
\end{aligned}
$$

with $\boldsymbol{\tau}^{2}\left(y_{\beta}\right)$ known from Eq. (64) and $\partial_{3} \mathbf{e}^{1}\left(x_{3}\right)$ from Eq. (101) and (102). $\boldsymbol{\sigma}^{2}\left(y_{\beta}, x_{3}\right)$ in Eq. (94) becomes:

$$
\begin{aligned}
\boldsymbol{\sigma}^{2}\left(y_{\beta}, x_{3}\right)= & \boldsymbol{\tau}^{2}\left(y_{\beta}\right) \cdot \partial_{3} \mathbf{e}^{1}\left(x_{3}\right) \\
= & \left\{G\left[v \Phi_{11}+v \partial_{1} \theta_{1}+(1+v) \partial_{1} \eta_{1}\right]\left(\mathbf{e}_{\mathbf{1}} \otimes \mathbf{e}_{3}+\mathbf{e}_{3} \otimes \mathbf{e}_{\mathbf{1}}\right)\right. \\
& \left.+G\left[v \Phi_{21}+v \partial_{2} \theta_{1}+(1+v) \partial_{2} \eta_{1}\right]\left(\mathbf{e}_{2} \otimes \mathbf{e}_{3}+\mathbf{e}_{3} \otimes \mathbf{e}_{2}\right)\right\} \cdot\left(-\frac{T_{1_{L}}^{\varepsilon}}{\left.E I_{1}^{\varepsilon}\right)}\right)
\end{aligned}
$$

considering that $G=\frac{E}{2(1+v)}$, with $\Phi_{\alpha \beta}$ given in Eq. (53), $\theta_{\beta}\left(y_{1}, y_{2}\right)$ and $\eta_{\beta}\left(y_{1}, y_{2}\right)$ in Eq. (63).

The stress fiel is finall obtained as:

$$
\begin{aligned}
\boldsymbol{\sigma}^{\varepsilon}\left(y_{\beta}, x_{3}\right)= & \varepsilon \boldsymbol{\sigma}^{1}\left(y_{\beta}, x_{3}\right)+\varepsilon^{2} \boldsymbol{\sigma}^{2}\left(y_{\beta}, x_{3}\right) \\
= & \varepsilon\left\{-y_{1}\left(L-x_{3}\right) \frac{\left.T_{1_{L}}^{\varepsilon} \mathbf{e}_{3} \otimes \mathbf{e}_{3}\right\}}{I_{1}^{\varepsilon}}\right. \\
& +\varepsilon^{2} \frac{1}{2(1+v)}\left\{\left[v \Phi_{11}+v \partial_{1} \theta_{1}+(1+v) \partial_{1} \eta_{1}\right]\left(\mathbf{e}_{\mathbf{1}} \otimes \mathbf{e}_{3}+\mathbf{e}_{3} \otimes \mathbf{e}_{1}\right)\right. \\
& \left.+\left[v \Phi_{21}+v \partial_{2} \theta_{1}+(1+v) \partial_{2} \eta_{1}\right]\left(\mathbf{e}_{2} \otimes \mathbf{e}_{3}+\mathbf{e}_{3} \otimes \mathbf{e}_{2}\right)\right\} \cdot\left(-\frac{T_{1_{L}}^{\varepsilon}}{I_{1}^{\varepsilon}}\right) .
\end{aligned}
$$


We show hereafter how the asymptotic expansion solutions coincide with the SaintVenant solutions. For simplicity, the different stress components are considered separately.

Axial Stress In Eq. (105), $\sigma_{33}^{2}\left(\mathbf{y}, x_{3}\right)=0$ and therefore:

$$
\sigma_{33}^{\varepsilon}=\varepsilon \sigma_{33}^{1}\left(y_{\beta}, x_{3}\right)=\varepsilon\left(-y_{1}\right)\left(L-x_{3}\right) \frac{T_{1_{L}}^{\varepsilon}}{I_{1}^{\varepsilon}}=x_{1}\left(x_{3}-L\right) \frac{T_{1_{L}}^{\varepsilon}}{I_{1}^{\varepsilon}},
$$

which coincides with the Saint-Venant solution Eq. (20).

\section{Shear Stress}

a. Beam with arbitrary cross-section No analytical solutions exist for $\theta_{\alpha}\left(y_{1}, y_{2}\right)$ and $\eta_{\alpha}\left(y_{1}, y_{2}\right)$ in Eq. (105) for this case. Nevertheless, we can reformulate Eq. (105) to show that it is formally identical with the Saint-Venant solution.

Stress $\sigma_{13}^{\varepsilon} \quad$ Following Eq. (105), the shear stress $\sigma_{13}^{\varepsilon}$ is:

$$
\begin{aligned}
\sigma_{13}^{\varepsilon} & =\varepsilon^{2} \frac{1}{2(1+v)}\left[v \Phi_{11}+v \partial_{1} \theta_{1}+(1+v) \partial_{1} \eta_{1}\right]\left(-\frac{T_{1_{L}}^{\varepsilon}}{I_{1}^{\varepsilon}}\right) \\
& =\varepsilon^{2} \frac{1}{2(1+v)}\left\{\frac{1}{2} v\left(y_{2}^{2}-y_{1}^{2}\right)+\partial_{1}\left[-v \theta_{1}-(1+v) \eta_{1}\right]\right\} \frac{T_{1_{L}}^{\varepsilon}}{I_{1}^{\varepsilon}} .
\end{aligned}
$$

The problem here is that $\theta_{1}$ and $\eta_{1}$ are not exactly determined, they are just define by problem Eq. (63). Due to Eq. (63), however, it is found that the term $\left(-v \theta_{1}-(1+v) \eta_{1}\right)$ satisfies

$$
\begin{cases}\partial_{\alpha \alpha}\left[-v \theta_{1}-(1+v) \eta_{1}\right]=-2 y_{1} & \text { in } S, \\ \partial_{n}\left[-v \theta_{1}-(1+v) \eta_{1}\right]=v\left\{\frac{y_{1}^{2}-y_{2}^{2}}{2} n_{1}+y_{1} y_{2} n_{2}\right\} & \text { on } \partial S,\end{cases}
$$

and then $\sigma_{13}^{\varepsilon}$ can be written as follows with $\Phi_{11}$ given in Eq. (53):

$$
\sigma_{13}^{\varepsilon}=\frac{1}{2(1+v)}\left\{\frac{1}{2} v\left(\varepsilon^{2} y_{2}^{2}-\varepsilon^{2} y_{1}^{2}\right)+\varepsilon^{2} \partial_{1}\left[-v \theta_{1}-(1+v) \eta_{1}\right]\right\} \frac{T_{1_{L}}^{\varepsilon}}{I_{1}^{\varepsilon}},
$$

where $\left(-v \theta_{1}-(1+v) \eta_{1}\right)$ satisfie Eq. (107).

Proof See Eqs. (15) and (107), and assume that the term $\zeta_{1}^{\varepsilon}\left(x_{1}, x_{2}\right)=\varepsilon^{p}\left(-v \theta_{1}-(1+v) \eta_{1}\right)$ Then it can be found that:

$$
\begin{aligned}
\partial_{\alpha \alpha} \zeta_{1}^{\varepsilon}\left(x_{1}, x_{2}\right) & =\frac{1}{\varepsilon^{2}} \frac{\partial}{\partial y_{\alpha} \partial y_{\alpha}}\left(\varepsilon^{p}\left(-v \theta_{1}-(1+v) \eta_{1}\right)\right)=\varepsilon^{p-2}\left(-2 y_{1}\right) \\
& =\varepsilon^{p-3}\left(-2 x_{1}\right)
\end{aligned}
$$

which equals Eq. $(15)_{1}$, if $p=3$. It is easy to fin that $p=3$ and therefore (representing $\left(-v \theta_{1}-(1+v) \eta_{1}\right)$ by $\left.\zeta_{1}\left(y_{1}, y_{2}\right)\right)$ :

$$
\zeta_{1}^{\varepsilon}\left(x_{1}, x_{2}\right)=\varepsilon^{3} \zeta_{1}\left(y_{1}, y_{2}\right)
$$


Consequently it is found that:

$$
\begin{aligned}
\partial_{1}^{\varepsilon} \zeta_{1}^{\varepsilon}\left(x_{1}, x_{2}\right) & =\frac{1}{\varepsilon} \frac{\partial}{\partial y_{1}} \zeta_{1}^{\varepsilon}\left(x_{1}, x_{2}\right)=\frac{1}{\varepsilon} \frac{\partial}{\partial y_{1}}\left(\varepsilon^{3} \zeta\left(y_{1}, y_{2}\right)\right) \\
& =\varepsilon^{2} \partial_{1}\left(-v \theta_{1}-(1+v) \eta_{1}\right)
\end{aligned}
$$

and in the same way we can get that:

$$
\partial_{2}^{\varepsilon} \zeta_{1}^{\varepsilon}\left(x_{1}, x_{2}\right)=\varepsilon^{2} \partial_{2}\left(-v \theta_{1}-(1+v) \eta_{1}\right) .
$$

Considering no warping $\varpi$, the shear stress of the Saint-Venant solution is given by Eq. (23) $)_{1}$. Taking into account that $\varepsilon y_{\alpha}=x_{\alpha}$, Eq. (108) and Eq. (23) $)_{1}$ are found to be identical.

Stress $\sigma_{23}^{\varepsilon}$ The asymptotic solution Eq. (105) becomes:

$$
\begin{aligned}
\sigma_{23}^{\varepsilon} & \left.=\varepsilon^{2} \frac{1}{2(1+v)}\left\{v \Phi_{21}+v \partial_{2} \theta_{1}+(1+v) \partial_{2} \eta_{1}\right]\right\}\left(-\frac{T_{1_{L}}^{\varepsilon}}{I_{1}^{\varepsilon}}\right) \\
& =\varepsilon^{2} \frac{1}{2(1+v)}\left\{-v y_{1} y_{2}+\partial_{2}\left[-v \theta_{1}-(1+v) \eta_{1}\right]\right\} \frac{T_{1_{L}}^{\varepsilon}}{I_{1}^{\varepsilon}}
\end{aligned}
$$

where $\left(-v \theta_{1}-(1+v) \eta_{1}\right)$ satisfie Eq. (107).

The Saint-Venant solution is given by Eq. $(23)_{2}$. As before, the consistency of the two solutions is obvious.

b. Beam with circular cross-section Analytical solutions for $\theta_{\alpha}\left(y_{1}, y_{2}\right)$ and $\eta_{\alpha}\left(y_{1}, y_{2}\right)$ are available for a circular cross section of radius $R$ :

$$
\left\{\begin{array}{l}
\theta_{\alpha}=-\frac{1}{4} y_{\alpha}\left(y_{1}^{2}+y_{2}^{2}-\varepsilon^{-2} R^{2}\right), \\
\eta_{\alpha}=\frac{1}{4} y_{\alpha}\left(y_{1}^{2}+y_{2}^{2}-3 \varepsilon^{-2} R^{2}\right) .
\end{array}\right.
$$

$\boldsymbol{\sigma}^{2}\left(y_{\beta}, x_{3}\right)$ in Eq. (105) is determined and the global stress solution for a beam with circular cross-section is completely obtained:

$$
\begin{aligned}
\boldsymbol{\sigma}^{\varepsilon}\left(y_{\beta}, x_{3}\right)= & \varepsilon \boldsymbol{\sigma}^{1}\left(y_{\beta}, x_{3}\right)+\varepsilon^{2} \boldsymbol{\sigma}^{2}\left(y_{\beta}, x_{3}\right)=\varepsilon\left[\begin{array}{ccc}
0 & 0 & 0 \\
& 0 & 0 \\
s y m & \left(-y_{1} E\right) \frac{T_{1_{L}}^{\varepsilon}\left(L-x_{3}\right)}{E I_{1}^{\varepsilon}}
\end{array}\right] \\
& +\varepsilon^{2}\left[\begin{array}{ccc}
0 & 0 & -\frac{1}{8}\left[(2 v+3)\left(y_{1}^{2}-\varepsilon^{-2} R^{2}\right)+(1-2 v) y_{2}^{2}\right] \\
& 0 & -\frac{1}{4}(1+2 v) y_{1} y_{2} \\
s y m & 0
\end{array}\right] \frac{T_{1_{L}}^{\varepsilon}}{(1+v) I_{1}^{\varepsilon}} .
\end{aligned}
$$

Considering that $\varepsilon y_{\alpha}=x_{\alpha}$ :

$$
\boldsymbol{\sigma}^{\varepsilon}\left(y_{\beta}, x_{3}\right)=\left[\begin{array}{ccc}
0 & 0 & -\frac{1}{8(1+v)}\left[(2 v+3)\left(x_{1}^{2}-R^{2}\right)+(1-2 v) x_{2}^{2}\right] \\
& 0 & -\frac{(1+2 v)}{4(1+v)} x_{1} x_{2} \\
s y m & & -x_{1}\left(L-x_{3}\right)
\end{array}\right] \frac{T_{1_{L}}^{\varepsilon}}{I_{1}^{\varepsilon}},
$$

which coincides with the Saint-Venant solution (Eqs. (20) and (25)). 
Fig. 4 Section and shear center

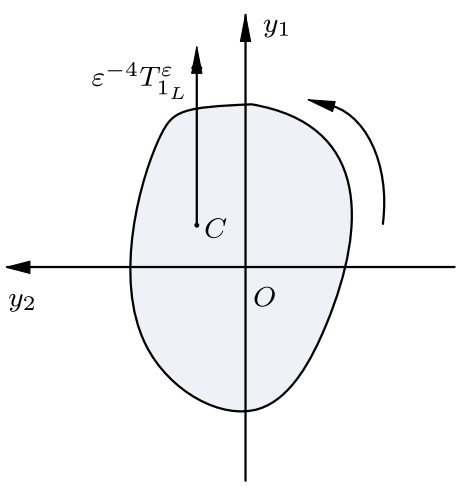

\subsubsection{Simple Bending Problem with Torsion}

According to Eq. (16), torsion is generally composed of three components due to moment and shear forces. For the simple bending problem, only torsion due to shear forces exists.

As already mentioned in Sect. 3, the shear center $C\left(y_{1 C}, y_{2 C}\right)$ may be located not on the beam axis (see Fig. 4). Then the shear force $\varepsilon^{-4} T_{1_{L}}^{\varepsilon}$ generates a moment of torsion given by:

$$
M_{3}^{1}(L)=0, \quad M_{3}^{2}(L)=-\varepsilon^{-4} T_{1_{L}}^{\varepsilon} y_{2 C},
$$

on the right beam end and

$$
M_{3}^{1}(0)=0, \quad M_{3}^{2}(0)=-\varepsilon^{-4} T_{1_{L}}^{\varepsilon} y_{2 C},
$$

on the left beam end. Therefore, the torsion moment of the structure is:

$$
M_{3}^{2}\left(x_{3}\right)=-\varepsilon^{-4} T_{1_{L}}^{\varepsilon} y_{2 C} .
$$

Since the problems related to $M_{\alpha}^{1}\left(x_{3}\right)$ are already solved in the previous sections, only the torsion problem is studied hereafter.

According to the macroscopic constitutive law Eqs. (70) and (84) with respect to $M_{3}^{2}$ and the analytical solution in Eq. (73), considering also that $J^{\varepsilon}=\varepsilon^{4} J$, we get:

$$
\partial_{3} \varphi^{1}\left(x_{3}\right)=0, \quad \partial_{3} \varphi^{2}\left(x_{3}\right)=\frac{M_{3}^{2}\left(x_{3}\right)}{G J}=-\varepsilon^{-1} \frac{T_{1_{L}}^{\varepsilon} x_{2 C}}{G J^{\varepsilon}} .
$$

According to Eq. (94), the stress due to torsion $\sigma^{1 T}$ is obtained as:

$$
\boldsymbol{\sigma}^{1 T}\left(y_{\beta}, x_{3}\right)=\mathbf{0}, \quad \boldsymbol{\sigma}^{2 T}=\boldsymbol{\tau}^{1 T}\left(y_{\beta}\right) . \partial_{3} \varphi^{2}\left(x_{3}\right),
$$

with $\boldsymbol{\tau}^{1 T}$ given in Eq. (55):

$$
\begin{aligned}
\boldsymbol{\sigma}^{T \varepsilon} & =\varepsilon^{2} \boldsymbol{\tau}^{2 T}\left(y_{1}, y_{2}\right) . \partial_{3} \varphi^{2}\left(x_{3}\right) \\
& =\varepsilon^{2} G\left[\partial_{2} \Psi\left(\mathbf{e}_{\mathbf{1}} \otimes \mathbf{e}_{3}+\mathbf{e}_{\mathbf{3}} \otimes \mathbf{e}_{\mathbf{1}}\right)-\partial_{1} \Psi\left(\mathbf{e}_{\mathbf{2}} \otimes \mathbf{e}_{3}+\mathbf{e}_{3} \otimes \mathbf{e}_{2}\right)\right] . \partial_{3} \varphi^{2}\left(x_{3}\right) \\
& =\varepsilon G\left[\begin{array}{rrr}
0 & 0 & \partial_{1} \varpi\left(y_{1}, y_{2}\right)-y_{2} \\
& 0 & \partial_{2} \varpi\left(y_{1}, y_{2}\right)+y_{1} \\
s y m & 0
\end{array}\right]\left(-\frac{T_{1_{L}}^{\varepsilon} x_{2 C}}{G J^{\varepsilon}}\right) .
\end{aligned}
$$


By the same method as before, we can justify that: $\varpi^{\varepsilon}\left(x_{1}, x_{2}\right)=\varepsilon^{2} \varpi\left(y_{1}, y_{2}\right)$ and

$$
\partial_{\alpha}^{\varepsilon} \varpi^{\varepsilon}\left(x_{1}, x_{2}\right)=\varepsilon \partial_{\alpha} \varpi\left(y_{1}, y_{2}\right) .
$$

Then

$$
\boldsymbol{\sigma}^{T \varepsilon}=-\frac{T_{1_{L}}^{\varepsilon} x_{2 C}}{\boldsymbol{J}^{\varepsilon}}\left[\begin{array}{ccc}
0 & 0 & \partial_{1}^{\varepsilon} \varpi^{\varepsilon}\left(x_{1}, x_{2}\right)-x_{2} \\
& 0 & \partial_{2}^{\varepsilon} \varpi^{\varepsilon}\left(x_{1}, x_{2}\right)+x_{1} \\
s y m & & 0
\end{array}\right],
$$

which coincides with the torsion part of the Saint-Venant solution provided in Eq. (22).

Following a similar process, a coincidence between the asymptotic expansion solution and the Saint-Venant solution can also be found for the displacements.

\subsection{Conclusion}

It is shown in this article that the asymptotic expansion method provides identical solutions with the Saint-Venant 3D elastic solutions far from the beam edges for the case of homogeneous isotropic beams with arbitrary cross-sections. Actually, the asymptotic expansion solutions depend on microscopic solutions which are governed by the same equations as the warping solutions associated to the 3D elastic Saint-Venant solutions. The paper focused on stress solutions of pure and simple bending (coupling shear, torsion and bending) problems, but the approach can be easily applied for pure traction and torsion, as well as to fin the solutions in terms of displacements. It is also found that for the pure bending problems, the firs order microscopic solution suffices for the simple bending problem however, due to the shear effects, the second order solution is needed.

It would be interesting to extend this work to more complex cross-sections such as sandwich or laminated beams provided that analytical solutions are available.

Acknowledgements The authors gratefully acknowledge the financia support provided by the China Scholarship Council (CSC).

\section{References}

1. Bamberger, Y.: Mécanique de l'ingénieur III-solides déformables. Hermann étude des science et des arts, Paris (1997)

2. Berdichevsky, V.L.: Variational Principles of Continuum Mechanics. II. Applications. Springer, Berlin (2009)

3. Boutin, C.: Microstructural effects in elastic composites. Int. J. Solids Struct. 33(7), 1023-1051 (1996)

4. Buannic, N.: Analyse Asymptotique de Poutres Elastique Hétérogènes. Ph.D. thesis, Ecole Centrale de Nantes (2000)

5. Buannic, N., Cartraud, P.: Higher-order asymptotic model for a heterogeneous beam, including corrections due to end effects. In: AIAA, American Institute of Aeronautics and Astronautics, 41st Structures, Structural Dynamics, and Materials Conference and Exhibit. AIAA, Atlanta (2000)

6. Buannic, N., Cartraud, P.: Higher-order effective modeling of periodic heterogeneous beams. I. Asymptotic expansion method. Int. J. Solids Struct. 38(40-41), 7139-7161 (2001)

7. Buannic, N., Cartraud, P.: Higher-order effective modeling of periodic heterogeneous beams. II. Derivation of the proper boundary conditions for the interior asymptotic solution. Int. J. Solids Struct. 38, $7163-7180$ (2001)

8. Carrera, E., Petrolo, M.: On the effectiveness of higher-order terms in refine beam theories. J. Appl. Mech. 78(2), 021013 (2011)

9. Cimetière, A., Geymonat, G., Le Dret, H., Raoult, A., Tutek, Z.: Asymptotic theory and analysis for displacements and stress distribution in nonlinear elastic straight slender rods. J. Elast. 19(2), 111-161 (1988). doi:10.1007/BF00040890 
10. Dauge, M., Yosibash, Z.: Boundary layer realization in thin elastic three-dimensional domains and twodimensional hierarchic plate models. Int. J. Solids Struct. 37(17), 2443-2471 (2000)

11. Hodges, D.H., Atilgan, A.R., Cesnik, C.E., Fulton, M.V.: On a simplifie strain energy function for geometrically nonlinear behaviour of anisotropic beams. Compos. Eng. 2(5), 513-526 (1992)

12. Horgan, C.O.: Recent developments concerning saint-Venant's principle: a second update. Appl. Mech. Rev. 42(11), 295-303 (1989)

13. Karama, M., Afaq, K.S., Mistou, S.: Mechanical behaviour of laminated composite beam by the new multi-layered laminated composite structures model with transverse shear stress continuity. Int. J. Solids Struct. 40(6), 1525-1546 (2003)

14. Kim, J.S., Wang, K.W.: On the asymptotic boundary conditions of an anisotropic beam via virtual work principle. Int. J. Solids Struct. 48(16-17), 2422-2431 (2011). doi:10.1016/j.ijsolstr.2011.04.016

15. Kolpakov, A.G.: Calculation of the characteristics of thin elastic rods with a periodic structure. J. Appl. Math. Mech. 55(3), 358-365 (1991)

16. Kolpakov, A.G.: Stressed Composite Structures: Homogenized Models for Thin-Walled Nonhomogeneous Structures with Initial Stresses. Springer, Berlin (2004)

17. Marigo, J.J., Ghidouche, H., Sedkaoui, Z.: Des poutres fl xibles aux fil extensibles : une hiérarchie de modéles asymptotiques. C. R. Acad. Sci. 326(2), 79-84 (1998)

18. Marur, S.R., Kant, T.: Free vibration analysis of fibe reinforced composite beams using higher order theories and finit element modelling. J. Sound Vib. 194(3), 337-351 (1996)

19. Miara, B.: Justificatio des mises à l'échelle et des hypothèses sur les données dans l'analyse asymptotique des modèles bidimensionnels de plaques minces élastiques. I : Le cas non linéaire. C. R. Acad. Sci. 314(1), 687-690 (1992)

20. Sanchez-Hubert, J., Sanchez-Palencia, E.: Introduction aux méthodes asymptotiques et à l'homogénéisation. Masson, Paris (1992)

21. Trabucho, L., Viaño, J.M.: Mathematical modelling of rods. In: Ciarlet, P.G., Lions, J.L. (eds.) Handbook of Numerical Analysis, vol. IV, pp. 487-974. North-Holland, Amsterdam (1996)

22. Yu, W., Hodges, D.H.: Elasticity solutions versus asymptotic sectional analysis of homogeneous, isotropic, prismatic beams. J. Appl. Mech. 71(1), 15 (2004). doi:10.1115/1.1640367 